\title{
Prognostic-oriented Fuel Cell Catalyst Aging Modeling and Its Application to Health-Monitoring and Prognostics of a PEM Fuel Cell
}

\author{
Xian Zhang ${ }^{1}$, Pierluigi Pisu ${ }^{2}$ \\ ${ }^{1,2}$ Department of Automotive Engineering, Clemson University, Clemson, SC, 29631, USA \\ xianz@g.clemson.edu \\ pisup@clemson.edu
}

\begin{abstract}
Today, poor long-term performance and durability combined with high production and maintenance costs remain the main obstacles for the commercialization of the polymer electrolyte membrane (PEM) fuel cells (PEMFCs). While on-line diagnosis and operating condition optimization play an important role in addressing the durability issue of the fuel cell, health-monitoring and prognosis (or PHM) techniques are of equally great significance in terms of scheduling condition-based maintenance $(\mathrm{CBM})$ to minimize repair and maintenance costs, the associated operational disruptions, and also the risk of unscheduled downtime for the fuel cell systems.

The two essential components of a PHM scheme for a general engineering system are 1) an accurate aging model that is capable of capturing the system's gradual health deterioration, and 2) an algorithm for damage estimation and prognostics. In this paper, a physics-based, prognosticoriented fuel cell catalyst degradation model is developed to characterize the relationship between the operating conditions and the degradation rate of the electro-chemical surface area (ECSA). The model complexity is kept minimal for on-line prognostic purpose. An unscented Kalman filter (UKF) approach is then proposed for the purpose of damage tracking and remaining useful life prediction of a PEMFC.
\end{abstract}

\section{INTRODUCTION}

To date long-term performance and durability of the fuel cells are difficult to quantify because not all degradation mechanisms of the various fuel cell components are completely understood. The fuel cell's performance degrades irreversibly throughout its lifetime mainly due to the following components' degradations: (1) catalyst degradation (catalyst particle coarsening); (2) carbon support degradation (carbon corrosion); and (3) membrane degradation (chemical deterioration and dehydration) (Okada, 2003; Schmittinger \& Vahidi, 2008). Factors affecting these degradation processes include temperature, high potentials, heat cycle but most of all water management, contaminants, and impurities. Thus, depending on the power load and the long-term operating conditions of the fuel cell, the extent of performance and durability degradation varies. In general, the longer the fuel cell stack is operated in transient or cycling conditions, or detrimental operating conditions such as flooding, the stronger is the corrosion and therefore the degradation.

While extensive research has been carried out on control and dynamical modeling for fuel cell system (Alejandro, Arce, \& Bordons, 2007; Danzer, Wilhelm, Aschemann, \& Hofer, 2008; Miotti, Di Domenico, Guezennec, \& Rajagopalan, 2005; Pukrushpan, Peng, \& Stefanopoulou, 2004; Soltani \& Bathaee, 2010), modeling work addressing PEMFC degradation and corresponding health-monitoring and prognostic system has been much less reported (Zhang \& Pisu, 2012). Many of the degradation models have little physical basis, and thus have no predictive capability (A.A. Franco \& Tembely, 2007). Franco et al. (A. A. Franco, Schott, Jallut, \& Maschke, 2007; Alejandro A. Franco \& Gerard, 2008; Alejandro A. Franco et al., 2009; Alejandro A. Franco, Gerard, Guinard, Barthe, \& Lemaire, 2008) have done a series of leading work on developing a multi-scale mechanistic model of the electrochemical aging processes in a PEMFC to describe, in particular, the carbon corrosion at the cathode, the cathodic oxidation/dissolution of platinum and the carbon supported platinum electrochemical ripening. However, its computational fluid dynamics (CFD) modeling approach makes it unsuitable for the on-line prognostic purpose due to the computation burden involved. Also, the model needs too many parameters that are hard to obtain. Darling and Meyers (R.M. Darling \& Meyers, 2003) proposed a spatially lumped model that treats a single, porous platinum electrode and the ionomeric solution that fills the pores of the electrode. The model includes spherical platinum particles that can grow and shrink as platinum plates and dissolves, a platinum oxide layer, and an ionic platinum species in solution $\left(\mathrm{Pt}^{2+}\right)$. The kinetic expressions for platinum oxidation and dissolution developed in this work is incorporated by the same authors (Robert M. Darling \& Meyers, 2005) in a transient, one-dimensional 
mathematical model of the cross section of a PEM fuel cell. In this model, each electrode contains two platinum particle sizes, enabling a description of electrochemically driven transfer of platinum between particles of different sizes. That is, platinum can be exchanged between particles by dissolution and crystallization, capturing the underlying principles of the quasi-Ostwald ripening. Simulation results, however, didn't quite capture the curvature of the ECSA evolution with time as seen in the durability tests, and show that the ECSA decreases linearly. The authors presumed that this may have been caused by short simulation time.

Bi and Fuller (Bi \& Fuller, 2008) modified the model in (R.M. Darling \& Meyers, 2003; Robert M. Darling \& Meyers, 2005) by considering the diffusion of Pt ions in the membrane electrode assembly (MEA), while still adopted the two particle groups model structure. The authors concluded that the model is not adequate to predict well the catalyst degradation rates including Pt nanoparticle growth, catalyst surface area loss and cathode Pt mass loss, and suggest other degradation mechanisms such as new $\mathrm{Pt}$ cluster formation on carbon support and neighboring $\mathrm{Pt}$ clusters coarsening be investigated.

Holby et al. (Edward F. Holby, Sheng, Shao-Horn, \& Morgan, 2009) investigated the influence of particle size distribution (PSD) and crossover hydrogen on the $\mathrm{Pt}$ nanoparticle stability in PEM fuel cells by extending the previous degradation model of Darling and Meyers (R.M. Darling \& Meyers, 2003; Robert M. Darling \& Meyers, 2005) to include PSD effects, more complete interfacial thermodynamics, and hydrogen crossover effects. With the PSD sampled non-uniformly at 700 distinct radii (with 200 radii concentrated in the small particle region of the final distribution for accuracy), the model successfully captures the evolution trend of the PSD and the ECSA of the catalyst with aging. However, considering so many particle groups also compromises the computational efficiency of the model.

In this paper, a physics-based, prognostic-oriented catalyst degradation model is developed to characterize the fuel cell damage that establishes the relationship between the operating conditions and the degradation rate of the electrochemical surface area (ECSA). Our approach follows from the analysis of the underlying principle of the catalyst degradation, investigation of the interacting behavior of multi-group particles of the catalyst and the effect of the particle size distribution, and analytical derivation of a reduced order model that can describe and predict the lumped ECSA based on the analysis result. The model complexity is thus kept minimal and is suitable for on-line prognostic purpose. The model is then utilized in a novel UKF framework for the damage tracking and RUL estimation of the PEM fuel cell.

The rest of the paper is organized as follows: Section 2 presents the derivation process of a prognostic-oriented fuel cell catalyst degradation model; Section 3 gives a brief introduction to the UKF framework; and in Section 4, we apply the UKF approach to the damage tracking and RUL prediction of the fuel cell, and discuss the results obtained from the simulation tests..

\section{Aging Modeling for Catalyst Degradation Characterization}

\subsection{Catalyst Degradation Mechanisms}

The main degradation mechanisms for the catalyst can be roughly divided into three steps.

The first step of this process is Pt dissolution/oxidation, where Pt atoms are either oxidized to $\mathrm{Pt}$ ions and dissolve into the ionomer phase in the catalyst layer, or form an oxide film on the surface of the Pt particles. Platinum dissolution is described by reaction (1) in the forward direction.

$$
\mathrm{Pt} \stackrel{\nu_{1}}{\rightleftharpoons} \mathrm{Pt}^{2+}+2 \mathrm{e}^{-}
$$

$\mathrm{Pt}^{2+}$ is assumed to be the only ionic platinum species present in significant amounts, while other ionic species with higher oxidation states are not expected to be important in the region of interest (R.M. Darling \& Meyers, 2003).

Platinum oxide film formation and the subsequent dissolution of this film are described by (2) and (3) respectively.

$$
\begin{gathered}
\mathrm{Pt}+\mathrm{H}_{2} \mathrm{O} \stackrel{\nu_{2}}{\rightleftharpoons} \mathrm{PtO}+2 \mathrm{H}^{+}+2 \mathrm{e}^{-} \\
\mathrm{PtO}+2 \mathrm{H}^{+} \rightleftharpoons \mathrm{Pt}^{2+}+\mathrm{H}_{2} \mathrm{O}
\end{gathered}
$$

When exposed to high voltage, the formation of the oxide layer actually serves to protect the $\mathrm{Pt}$ particles from accelerated dissolution through (1) due to elevated potential across the interface of the electrolyte and the cathode. Although it is thermodynamically possible for the $\mathrm{PtO}$ to be further chemically dissolved through reaction (3), the kinetic rate of this reaction is considered to be extremely slow. Therefore, (3) is neglected in our degradation model.

The second step of the quasi-Ostwald process is the migration of $\mathrm{Pt}$ ions, where the dissolved $\mathrm{Pt}$ ions $\left(\mathrm{Pt}^{2+}\right)$ migrate to nearby $\mathrm{Pt}$ particles, with a distance of a few nanometers [17]. The ions can also migrate into the proton exchange membrane and precipitate there by reduction of $\mathrm{Pt}^{2+}$ by crossover $\mathrm{H}_{2}$ from anode, as described by (4).

$$
\mathrm{Pt}^{2+}+\mathrm{H}_{2} \rightleftharpoons \mathrm{Pt}+2 \mathrm{H}^{+}
$$

This reaction results in the absolute mass loss of the effective catalyst and the forming of a Pt "band" in the membrane (Bi \& Fuller, 2008). The Pt band is actually observed by electron probe microanalysis (EPMA) inside the electrolyte membrane of the used MEA at a location 
near the cathode catalyst layer (Shimoi, Aoyama, \& Iiyama, 2009). However, the authors in (Shimoi et al., 2009) also reported that the amount of $\mathrm{Pt}$ inside the electrolyte membrane was markedly smaller than the Pt loading of the cathode catalyst layer. Another evidence of the negligibility of the Pt precipitation in the membrane can be found in (Debe, Schmoeckel, Vernstrom, \& Atanasoski, 2006), where the surface area loss in both catalyst types appears to be primarily by agglomeration. Based on these results, reaction (4) is not considered in this study.

The third step of the process is the $\mathrm{Pt}$ ions reduction and redeposition on the $\mathrm{Pt}$ particle surface, which is described by reaction (1) in the reverse direction. $\mathrm{Pt}^{2+}$ ions tend to redeposit on larger $\mathrm{Pt}$ particles that have lower surface energy. This can be seen in the kinetic rate equation for the $\mathrm{Pt}$ dissolution/deposition to be presented later.

Since the key kinetic reactions involved in this process, i.e., (1) and (2), are both electro-chemical reactions, it becomes obvious that potential load is the main driving force for the quasi-Ostwald ripening. In fact, during the potential rise, $\mathrm{Pt}$ dissolution, along with the Pt oxidation, is facilitated due to favored electro-chemical environment, with the smaller Pt particle dissolving in a faster rate; while when potential drops, Pt ion reduction is facilitated and tends to redeposit on larger $\mathrm{Pt}$ particle due to its lower surface energy. Repeated cycling of the potential therefore results in larger catalyst particles getting larger while smaller catalyst particles getting even smaller. The consequence is then $\mathrm{Pt}$ ripening, which in turn causes decreased ECSA.

The quasi-Ostwald ripening mechanism explains why the high potential and the load cycling are the main aging factors for catalyst degradation. There is, however, another mechanism for catalyst degradation - particle migration and coalescence - that is not driven by the potential. But due to the fact that the role of this mechanism in the Pt surface area loss at low-temperature fuel cells is still uncertain, and there still lacks unique experiment evidence in support of crystal migration and coalescence (Shao-Horn et al., 2007), it is not considered in the catalyst degradation modeling here.

\subsection{N-Group Catalyst Degradation Model}

In this subsection, we establish a detailed catalyst degradation model based on the platinum dissolution kinetic model proposed by Darling and Meyers in (R.M. Darling \& Meyers, 2003; Robert M. Darling \& Meyers, 2005). The main assumptions for this model are as follows:

1) The model is spatially lumped that treats a single porous platinum electrode, and the ionomeric solution that fills the pores of the electrode (R.M. Darling \& Meyers, 2003), while the concentration gradient of the Pt ion across the catalyst layer is neglected.

2) Pt precipitation in the membrane is neglected.
3) The cathode includes Pt/C solid phase uniformly mixed with Nafion electrolyte and gas pore phase. The polymer (and/or ionomer) volume fractions were assumed to be $30 \%$ in the cathode and $100 \%$ in Nafion membrane (Bi \& Fuller, 2008).

4) There are $N$ groups of Pt particles. All particles in each group have a unique and identical initial radius at the fresh state of the MEA, and they are uniformly distributed across the cathode.

5) The particle can grow and shrink during the aging process, but the particle number in each group remains the same; also, the sizes of all the particles in a group are the same at all times.

6) When the radius of one group of Pt particles reached the $\mathrm{Pt}$ atom radius, that group of $\mathrm{Pt}$ particles is considered to be dissolved completely into the inomer phase.

7) PtO grows as a film of uniform thickness, on the surface of the platinum crystallites.

Now, for particles in group $i$, we can obtain the dynamics of the characteristic variable, i.e., the radius $r_{i}$ and the $\mathrm{PtO}$ coverage $\theta_{\mathrm{PtO}}^{(i)}$, by material balance, which are given in (5) (R.M. Darling \& Meyers, 2003)

$$
\left\{\begin{array}{c}
\frac{d r_{i}}{d t}=-\frac{M_{\mathrm{Pt}}}{\rho_{\mathrm{Pt}}} v_{1}^{(i)} \\
\frac{d \theta_{\mathrm{PtO}}^{(i)}}{d t}=\frac{v_{2}^{(i)}}{\Gamma_{\max }}-\frac{2 \theta_{\mathrm{PtO}}^{(i)}}{r_{i}} \frac{d r_{i}}{d t}
\end{array}\right.
$$

where $M_{\mathrm{Pt}}$ and $\rho_{\mathrm{Pt}}$ are the molecular weight and density of platinum, respectively. $v_{1}$ and $v_{2}$ refer to the reaction rates of (1) and (2), in moles per second per square centimeter of Pt surface area. $\Gamma_{\max }$ is the number of moles of active sites per unit of platinum area. This number is taken to be constant (R.M. Darling \& Meyers, 2003) and is calculated assuming a specific charge of $220 \mu \mathrm{C} / \mathrm{cm}^{2}$ in the hydrogen adsorption region. This equation indicates that $\mathrm{PtO}$ grows as a film of uniform thickness, on the surface of the platinum crystallites.

The reaction rate $v_{1}$ for the $\mathrm{Pt}$ dissolution rate $\left(\mathrm{Pt} \rightleftharpoons \mathrm{Pt}^{2+}+2 \mathrm{e}^{-}\right)$is expressed in (6)

$$
v_{1}^{(i)}=k_{1} \theta_{\mathrm{vac}}^{(i)}\left\{\begin{array}{l}
\exp \left[\frac{F}{R T}\left(\Delta \phi_{c}-U_{1}^{\theta}+\frac{\alpha_{1}}{r_{i}}\right)\right] \\
\left.-\left(C_{\mathrm{Pt}^{2+}} / C_{\mathrm{Pt}^{2+}}^{\mathrm{ref}}\right) \cdot \exp \left[-\frac{F}{R T}\left(\Delta \phi_{c}-U_{1}^{\theta}+\frac{\alpha_{1}}{r_{i}}\right)\right]\right\}
\end{array}\right\}
$$

where $k_{1}$ is a constant characteristic of the kinetic rate of the reaction, and is usually determined by experiments; $F$ is the 
Faraday constant, $R$ is the universal gas constant $(\mathrm{J} / \mathrm{mol} \cdot \mathrm{K})$ and $T$ the absolute temperature $(\mathrm{K}) ; \Delta \phi_{c}$ is the phase potential difference between the electrolyte phase and the cathode phase; $U_{1}^{\theta}=1.188 \mathrm{~V}$ is the standard equilibrium potential of reaction (1) for bulk platinum; $\alpha_{1}=1.14 \times 10^{-10} ; \theta_{\text {vac }}^{(i)}$ is the fraction of the platinum surface that is not covered by oxides, and $\theta_{\mathrm{vac}}^{(i)}=\max \left(0,1-\theta_{\mathrm{PtO}}^{(i)}\right)$, due to the fact that the PtO film can grow beyond one layer. $C_{\mathrm{Pt}^{2+}}$ and $C_{\mathrm{Pt}^{2+}}^{\text {ref }}$ are the actual and reference concentration for $\mathrm{Pt}^{2+}\left(C_{\mathrm{Pt}^{2+}}^{\text {ref }}\right.$ is chosen to be $1 \mathrm{M}$, i.e., $1 \mathrm{~mol} / \mathrm{L}$ in this work $)$ while $C_{\mathrm{P}^{2+}}$ can be written as (7) using mass balance.

$$
\frac{d C_{\mathrm{Pt}^{2+}}}{d t}=\frac{\sum_{i} 4 \pi r_{i}^{2} N_{i} v_{1}^{(i)}}{A_{\mathrm{fc}} \cdot \delta_{\mathrm{CL}}}
$$

where $N_{i}$ is the particle number in the $i$-th group. $A_{\mathrm{fc}}$ and $\delta_{\mathrm{CL}}$ are the nominal fuel cell area and the catalyst layer thickness, respectively.

The reaction rate $v_{2}$, the $\mathrm{Pt}$ oxidization rate $\left(\mathrm{Pt}+2 \mathrm{H}_{2} \mathrm{O} \rightleftharpoons \mathrm{PtO}+2 \mathrm{H}^{+}+2 \mathrm{e}^{-}\right)$is expressed in (8)

$$
v_{2}^{(i)}=k_{2}\left\{\begin{array}{l}
\exp \left(-\frac{\omega \theta_{\mathrm{PIO}}^{(i)}}{R T}\right) \exp \left[\frac{0.7 F}{R T}\left(\Delta \phi_{c}-\tilde{U}_{2}^{\theta}+\frac{\alpha_{2}}{r_{i}}\right)\right] \\
-\theta_{\mathrm{PtO}}\left(C_{\mathrm{H}^{+}} / C_{\mathrm{H}^{+}}{ }_{\mathrm{ref}}\right. \\
\left.{ }^{2} \cdot \exp \left[-\frac{0.3 F}{R T}\left(\Delta \phi_{c}-\tilde{U}_{2}^{\theta}+\frac{\alpha_{2}}{r_{i}}\right)\right]\right\}
\end{array}\right\}
$$

where $k_{2}$ is kinetic rate coefficient of the reaction; $C_{\mathrm{H}^{+}}$and $C_{\mathrm{H}^{+}}^{\text {ref }}$ are the actual and reference concentration for $\mathrm{H}^{+} ; \omega=30 \mathrm{~kJ} / \mathrm{mol}$ (R.M. Darling \& Meyers, 2003), $\tilde{U}_{2}^{\theta}=0.76 \mathrm{~V}$, and $\alpha_{2}=3.65 \times 10^{-11}$.

From the kinetic rate equations presented above, it now becomes clear that particles with smaller radius $r_{i}$ tend to have higher dissolution rate $v_{1}$ and oxidation rate $v_{2}$ under the same potential load.

It should be noted that, instead of the cell voltage measured across the fuel cell terminals, the aging process is actually driven by the phase potential difference between the electrolyte phase and the electrode phase, i.e., $\Delta \phi_{\mathrm{c}}=\phi_{\mathrm{c}}-\phi_{\mathrm{e}}$ in the case of cathode, where $\phi_{\mathrm{e}}$ is the electrical potential of the electrolyte, and $\phi_{\mathrm{c}}$ is the electrical potential at the cathode. As the cell voltage can be expressed as $V_{\text {cell }}=\Delta \phi_{\mathrm{c}}+\Delta \phi_{\mathrm{a}}-i \cdot A_{f c} \cdot R_{\text {ohm }}$, and the anode phase potential difference $\Delta \phi_{\mathrm{a}}=\phi_{\mathrm{e}}-\phi_{\mathrm{a}}$ can be neglected, the potential load can be obtained as $\Delta \phi_{\mathrm{c}}=V_{\text {cell }}+i \cdot A_{f c} \cdot R_{\text {ohm }}$, i.e., the so called "iR free voltage".

To simplify the notation, we define $\quad \exp \left(\frac{F}{R T} \Delta \phi_{c}\right)=u_{c}, \quad \exp \left(\frac{F}{R T} U_{1}^{\theta}\right)=u^{\theta} \quad$, and $\exp \left(\frac{F}{R T} \frac{\alpha_{1}}{r_{i}}\right)=f_{1}\left(r_{i}\right)$. It follows from (8) that

$$
v_{1}^{(i)}=k_{1} \theta_{\mathrm{vac}}^{(i)}\left\{\frac{u_{c}}{u^{\theta}} \cdot f_{1}\left(r_{i}\right)-\left(C_{\mathrm{Pt}^{2+}} / C_{\mathrm{Pt}^{2+}}^{\text {ref }}\right) \cdot \frac{u^{\theta}}{u_{c} f_{1}\left(r_{i}\right)}\right\}
$$

Also, we define $A_{\text {geo }}=\sum_{i} 4 \pi r_{i}^{2} N_{i}$ to be the geometric surface area of the catalyst, which is calculated by totalizing the surface area of all particle groups. Note that $A_{\text {geo }}$ does not equal to the ECSA, the two area values can be thought to be proportional to each other, with a typical rescaling coefficient of ECSA/ $A_{\text {geo }}=0.63$, as given by (Edward F. Holby et al., 2009).

As a first step to study the model behavior, we assume that only two particle groups are present in the cathode. The concept of the bimodal particle size distribution is also proposed in (Robert M. Darling \& Meyers, 2005).

For reaction rate coefficient, we choose $k_{1}=10^{-10}$ $\mathrm{mol} / \mathrm{cm}^{2} / \mathrm{s}, k_{2}=3 \times 10^{-10} \mathrm{~mol} / \mathrm{cm}^{2} / \mathrm{s}$, these rate parameters will be used throughout this section unless otherwise specified. Simulation result for the 2-group case with a constant potential load $\Delta \phi_{c}=0.97 \mathrm{~V}$ is shown in Figure 1 . The ratio of ECSA over nominal fuel cell area ECSA/ $A_{f c}$ is termed as catalyst magnifying coefficient here.
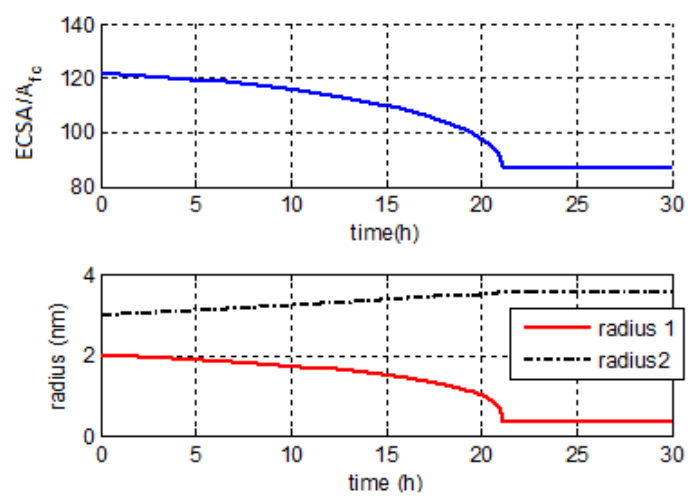

Figure 1. Evolution of the catalyst magnifying coefficient and the two radius with time (constant $0.97 \mathrm{~V}$ )

As seen from Figure 1, with the 2-particle group model, the catalyst magnifying coefficient (or equivalently the ECSA) decreases, due to the underlying ripening mechanism. But unfortunately, the model fails to capture the exponential-like shape of the ECSA decay as observed from experimental 
results as shown in (E.F. Holby, Shao-Horn, Sheng, \& Morgan, 2010). In fact, instead of having a degradation rate that gradually slows down, the modeled degradation rate in Figure 1 is accelerating throughout the decay process, and after about $21 \mathrm{~h}$, the ECSA reached a stagnant state.

Next, we assume that there are 64 particle groups in the cathode. Generally speaking, more particle groups would result in better model accuracy, but bigger computational burden at the same time. The number of 64 is chosen here to balance both the accuracy and the computational burden.

A normal distribution for the particle size is assumed in the initial state; the bell shaped distribution plot is shown in the following. The simulation is carried out with the same constant potential load $\Delta \phi_{c}=0.97 \mathrm{~V}$. Simulation results are presented in Figure $3 \sim$ Figure 5.

In Figure 3, it can be seen that the 64-particle-size model has now successfully captured the exponential trend of the ECSA decay that is observed in the PEM fuel cell aging test. Figure 4 shows the evolution of the particles size distribution during aging at $100 \mathrm{~h}, 200 \mathrm{~h}, 300 \mathrm{~h}$, and $700 \mathrm{~h}$ respectively. The plots qualitatively reflect the trend of the particle size distribution to spread more widely and shift to larger particle size region. Figure 5 depicts the radius evolution of all particle groups with aging, with the mean radius plotted in bold blue line. It can be seen that the smaller groups dissolve and disappear one by one (indicated by crossing the green horizontal line that represents the $\mathrm{Pt}$ atom radius), while the larger particles grow at the cost of these small particle size groups.

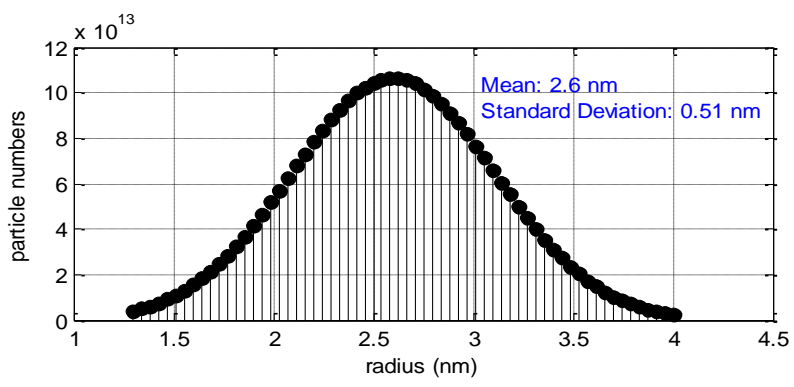

Figure 2. Particle size distribution in the initial state

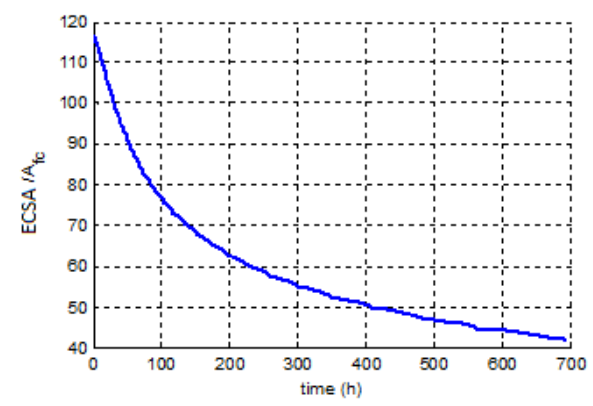

Figure 3 . The evolution of the catalyst magnifying coefficient for 64-particle model (constant $0.97 \mathrm{~V}$ )
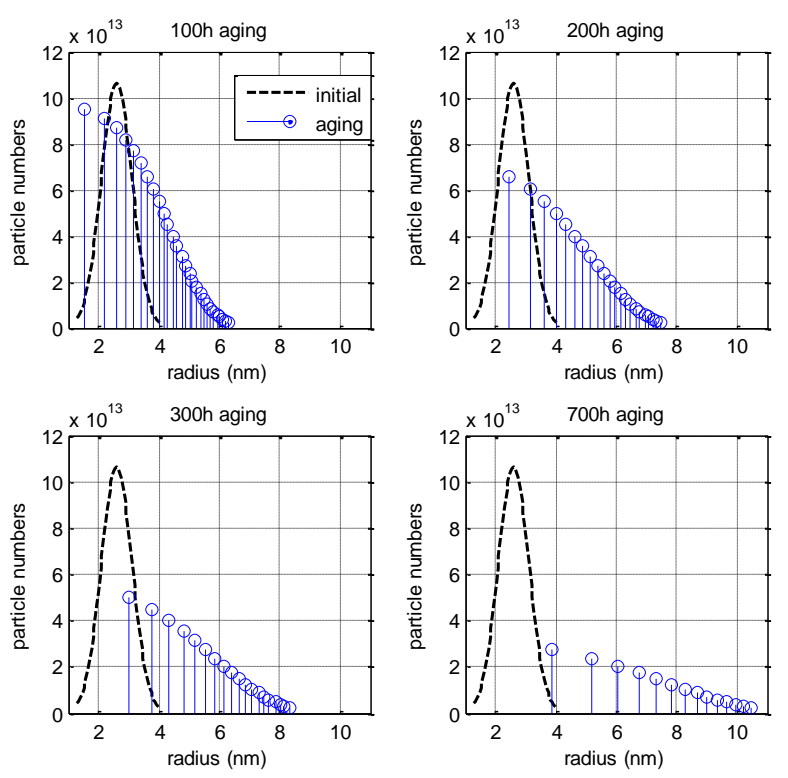

Figure 4. Evolution of the particles size distribution during aging (constant $0.97 \mathrm{~V}$ )

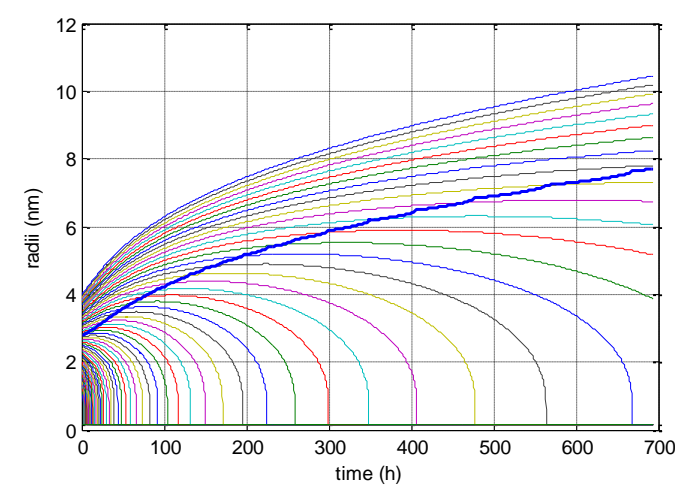

Figure 5. Radius evolution of all particle groups with time (constant $0.97 \mathrm{~V}$ )

It is now clear that with sufficient partitioning of the particle groups of the catalyst, the electrochemically driven dissolution and re-deposition catalyst ripening mechanism can well explain and capture the exponential decay of the ECSA damage variable. However, in terms of on-line prognostic, fine partitioning of the catalyst particle model also imposes a great computational burden on the prognostic scheme and thus considered to be unsuitable for this purpose. Next, by analysis of the underlying principle of the catalyst degradation, investigation of the interacting behavior of multi-group particles of the catalyst and the effect of the particle size distribution, we analytically derive a reduced order aging model that not only keeps the model complexity minimal, but is also capable of describing and predicting the lumped ECSA. 


\subsection{Model Simplification for Prognostic Purpose}

Before deriving the simplified degradation model, we analyze 2-particle-size model in more details. Simulation is carried out using a cycling potential load with $0.8 \mathrm{~V}-1 \mathrm{~V}$ square profile, as shown in Figure 6. Simulation results are presented in Figure 7 Figure 9.

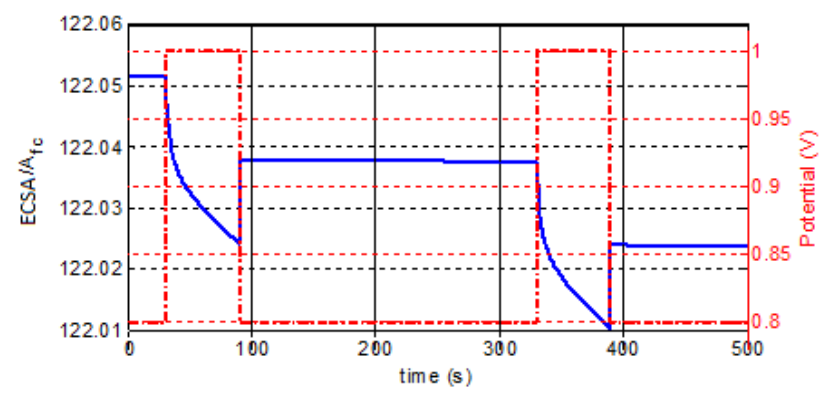

Figure 6 . The evolution of the catalyst magnifying coefficient for 2-particle-size model (cycling potential with square profile, $0.8 \mathrm{~V}-1 \mathrm{~V}$ )
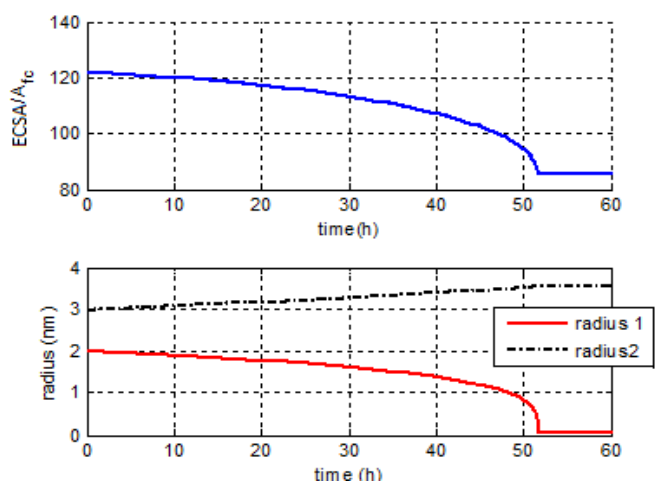

Figure 7. Evolution of the catalyst magnifying coefficient and the two radius with time (cycling potential with square profile, $0.8 \mathrm{~V}-1 \mathrm{~V}$ )
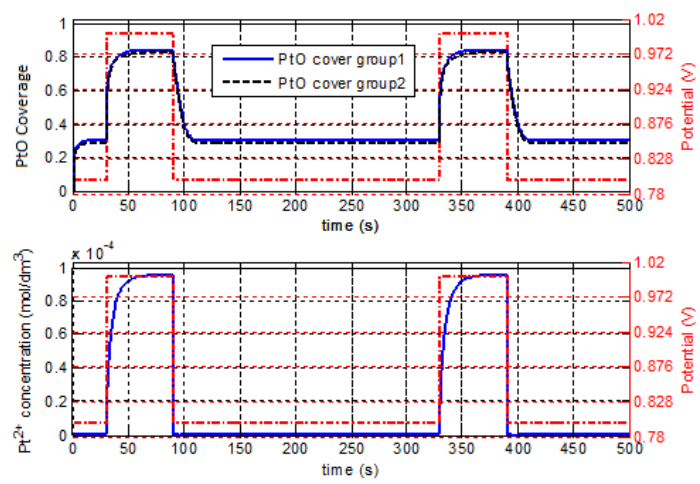

Figure 8. PtO coverage of the two groups and the $\mathrm{Pt}$ ion concentration with time (cycling potential with square profile, $0.8 \mathrm{~V}-1 \mathrm{~V}$ )

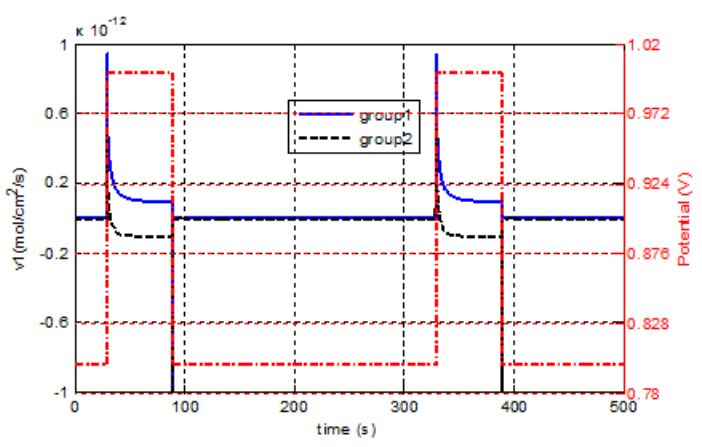

Figure 9 Dissolution rate for the two groups with time (cycling potential with square profile, $0.8 \mathrm{~V}-1 \mathrm{~V}$ )

In Figure 7, ECSA (equivalent to the catalyst magnifying coefficient) is seen to have a much greater degradation rate at high potential, i.e., $1 \mathrm{~V}$ hold, in contrast to the $0.8 \mathrm{~V}$ hold. This can be better illustrated by Figure 9, where it is seen that, during the $1 \mathrm{~V}$ hold, Pt particles with smaller size (group 1) has a positive dissolution rate while Pt particles with larger size (group 2) has a negative dissolution rate, meaning the $\mathrm{Pt}$ dissolution and $\mathrm{Pt}$ ion redeposition are actually occurring at the same time even if the potential load is hold constant.

From Figure 8, we can see the Pt ion concentration reaches its steady state about 20 s after the potential rise. The steady state is possible only when the number of dissolving Pt atom per unit time equals the number of redepositing $\mathrm{Pt}$ ion per unit time. By referring to the Pt ion dynamics in (7), we have at steady state that

$$
\begin{gathered}
\frac{d C_{\mathrm{Pt}^{2+}}}{d t}=\frac{\sum_{i} 4 \pi r_{i}^{2} N_{i} v_{1}^{(i)}}{A_{\mathrm{fc}} \cdot \delta_{\mathrm{CL}}}=0 \Rightarrow \sum_{i} r_{i}^{2} N_{i} v_{1}^{(i)}=0 \\
\Rightarrow \sum_{i} r_{i}^{2} N_{i} k_{1} \theta_{\mathrm{vac}}^{(i)}\left\{\begin{array}{l}
\frac{u_{c}}{u^{\theta}} \cdot f_{1}\left(r_{i}\right) \\
\left.-\left(C_{\mathrm{Pt}^{2+}} / C_{\mathrm{Pt}^{2+}}^{\mathrm{ref}}\right) \cdot \frac{u^{\theta}}{u_{c} f_{1}\left(r_{i}\right)}\right\}
\end{array}\right\}=0 \\
\Rightarrow k_{1}\left\{\begin{array}{l}
\frac{u_{c}}{u^{\theta}} \sum_{i} r_{i}^{2} N_{i} \theta_{\mathrm{vac}}^{(i)} \cdot f_{1}\left(r_{i}\right) \\
\left.-\left(C_{\mathrm{Pt}^{2+}} / C_{\mathrm{Pt}^{2+}}^{\mathrm{ref}}\right) \frac{u^{\theta}}{u_{c}} \sum_{i} r_{i}^{2} N_{i} \theta_{\mathrm{vac}}^{(i)} \cdot \frac{1}{f_{1}\left(r_{i}\right)}\right\}=0
\end{array}\right.
\end{gathered}
$$

From the above equation, we then get the steady state of the $\mathrm{Pt}$ ion concentration corresponding to the equivalent potential input $u_{c}$ as

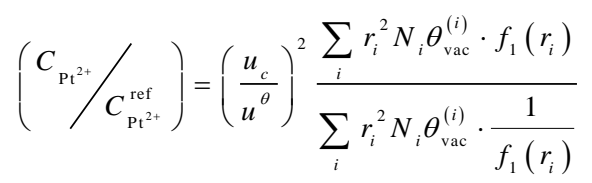




$$
=\left(\frac{u_{c}}{u^{\theta}}\right)^{2} \frac{\sum_{i} r_{i}^{2} N_{i} \theta_{\text {vac }}^{(i)} \cdot \frac{1}{f_{1}\left(r_{i}\right)} \cdot\left[f_{1}\left(r_{i}\right)\right]^{2}}{\sum_{i} r_{i}^{2} N_{i} \theta_{\text {vac }}^{(i)} \cdot \frac{1}{f_{1}\left(r_{i}\right)}} \triangleq\left(\frac{u_{c}}{u^{\theta}}\right)^{2}\left[f_{1}\left(r_{\text {avg }}\right)\right]^{2}
$$

where $r_{\text {avg }}$ is the equivalent average radius defined by the above equation. Note that the steady state $\mathrm{Pt}$ ion concentration is independent with the kinetic reaction rate $k_{1}$, while a function of the potential load and the average radius of the Pt particles.

Now revisit the dissolution rate $v_{1}^{(i)}=k_{1} \theta_{\mathrm{vac}}^{(i)}\left\{\frac{u_{c}}{u^{\theta}} \cdot f_{1}\left(r_{i}\right)-\left(C_{\mathrm{Pt}^{2+}} / C_{\mathrm{Pt}^{2+}}^{\mathrm{ref}}\right) \cdot \frac{u^{\theta}}{u_{c} f_{1}\left(r_{i}\right)}\right\}$, and note that $f_{1}\left(r_{i}\right)=\exp \left(\frac{F}{R T} \frac{\alpha_{1}}{r_{i}}\right)$ is a decreasing function. It then becomes obvious that, at a certain level of $\mathrm{Pt}$ ion concentration, the smaller particles have higher dissolution rates. In fact, there theoretically exists a radius such that the dissolution rate $v_{1}=0$, i.e. the particles with this radius would neither dissolve nor be redeposited by the Pt ions. The radius, which is defined by us as balanced radius and denoted as $r_{\text {bal }}$, satisfies the following equation:

$$
\begin{gathered}
k_{1} \theta_{\text {vac }}^{(i)}\left\{\frac{u_{c}}{u^{\theta}} \cdot f_{1}\left(r_{\text {bal }}\right)-\left(C_{\mathrm{Pt}^{2+}} / C_{\mathrm{Pt}^{2+}}^{\text {ref }}\right) \cdot \frac{u^{\theta}}{u_{c} f_{1}\left(r_{\text {bal }}\right)}\right\}=0 \Rightarrow \\
\left(C_{\mathrm{Pt}^{2+}} / C_{\mathrm{Pt}^{2+}}^{\mathrm{ref}}\right)=\left(\frac{u_{c}}{u^{\theta}}\right)^{2}\left[f_{1}\left(r_{\text {bal }}\right)\right]^{2}
\end{gathered}
$$

By comparing the above equation to (10), it is clear that, $r_{\text {bal }}$ and $r_{\text {avg }}$ are equivalent with each other in the sense of Pt ion concentration balance. All the particles with smaller radius than the balanced radius have positive dissolution rate and shrink in size, while the other particles larger than the balanced radius have negative dissolution rate (redeposition) and grow.

Now, by taking the derivative of the geometric catalytic surface area $A_{\text {geo }}=\sum_{i} 4 \pi r_{i}^{2} N_{i}$ with respect to time, we have

$$
\begin{aligned}
\frac{d A_{\mathrm{geo}}}{d t} & =\sum_{i} \frac{d}{d t}\left(4 \pi r_{i}^{2} N_{i}\right)=\sum_{i} 4 \pi \cdot 2 r_{i} \cdot \dot{r}_{i} \cdot N_{i} \\
& =\sum_{i} 4 \pi \cdot 2 r_{i} \cdot\left(-\frac{M_{\mathrm{Pt}}}{\rho_{\mathrm{Pt}}} v_{1}^{(i)}\right) \cdot N_{i} \\
& =-8 \pi \cdot \frac{M_{\mathrm{Pt}}}{\rho_{\mathrm{Pt}}} \sum_{i} r_{i} \cdot v_{1}^{(i)} \cdot N_{i}
\end{aligned}
$$

Substituting (9) and (10) into the above equation, we get

$$
\begin{aligned}
& \frac{d A_{\mathrm{geo}}}{d t}=-8 \pi \cdot \frac{M_{\mathrm{Pt}}}{\rho_{\mathrm{Pt}}} \sum_{i} r_{i} \cdot v_{1}^{(i)} \cdot N_{i} \\
& =-8 \pi \cdot \frac{M_{\mathrm{Pt}}}{\rho_{\mathrm{Pt}}} \sum_{i} r_{i} N_{i} k_{1} \theta_{\mathrm{vac}}^{(i)}\left\{\begin{array}{l}
\frac{u_{c}}{u^{\theta}} \cdot f_{1}\left(r_{i}\right) \\
-\left(C_{\mathrm{Pt}^{2+}} / C_{\mathrm{Pt}^{2+}}\right) \cdot \frac{u^{\theta}}{u_{c} f_{1}\left(r_{i}\right)}
\end{array}\right\} \\
& =-8 \pi \cdot \frac{M_{\mathrm{Pt}}}{\rho_{\mathrm{Pt}}} \sum_{i} r_{i} N_{i} k_{1} \theta_{\text {vac }}^{(i)}\left\{\begin{array}{l}
\frac{u_{c}}{u^{\theta}} \cdot f_{1}\left(r_{i}\right) \\
-\left(\frac{u_{c}}{u^{\theta}}\right)^{2}\left[f_{1}\left(r_{\text {avg }}\right)\right]^{2} \cdot \frac{u^{\theta}}{u_{c} f_{1}\left(r_{i}\right)}
\end{array}\right\} \Rightarrow \\
& \frac{d A_{\text {geo }}}{d t}=-8 \pi \cdot \frac{M_{\mathrm{Pt}}}{\rho_{\mathrm{Pt}}} \cdot k_{1} \frac{u_{c}}{u^{\theta}} \sum_{i} r_{i} N_{i} \theta_{\mathrm{vac}}^{(i)}\left\{f_{1}\left(r_{i}\right)-\frac{\left[f_{1}\left(r_{\mathrm{avg}}\right)\right]^{2}}{f_{1}\left(r_{i}\right)}\right\}
\end{aligned}
$$

To establish a connection between the catalytic area $A_{\text {geo }}$ and the average particle radius $r_{\text {avg }}$, the following approximation is made

$$
r_{\mathrm{avg}} \approx \bar{r}_{\mathrm{a}}=\frac{3 \cdot V_{\mathrm{Pt}}}{A_{\mathrm{geo}}}
$$

where $\bar{r}_{\mathrm{a}}$ is the average radius in the sense of particle geometry, and is the mean radius plotted in Figure 5.

Now, to further simplify the degradation rate equation as expressed in (13), we make the following assumptions:

1) At any time during the aging process, there are two fictitious $\mathrm{Pt}$ particle groups with radius $r_{1}$ and $r_{2}$ that can represent the averaged effect of the Pt particles with smaller and larger radius than $\bar{r}_{\mathrm{a}}$, respectively $\left(r_{1}<\bar{r}_{\mathrm{a}}\right.$ and $\left.r_{2}>\overline{r_{\mathrm{a}}}\right)$;

2) The sum of the areas of the two fictitious Pt particle groups equals to the total catalytic area, $a_{1}+a_{2}=A_{\text {geo }}$;

3) By defining the difference between the averaged and the fictitious radius as $\Delta r_{1}=\bar{r}_{\mathrm{a}}-r_{1}$, and $\Delta r_{2}=r_{2}-\bar{r}_{\mathrm{a}}$, we assume that $\frac{\Delta r_{1}}{\bar{r}_{\mathrm{a}}}$ and $\frac{\Delta r_{2}}{\bar{r}_{\mathrm{a}}}$ are constant throughout the aging process.

Assumption 3) is made by observation from Figure 5 and is consistent with the fact that the particle size distribution is spreading wider during the aging process. By making this assumption the modeling process is greatly simplified, and the simulation results show that it is a valid approximation.

From assumption 1) and by observing that the values of $\theta_{\text {vac }}^{(i)}$ for particle groups with different radii are very close to 
each other (which can be seen in Figure 8), we can impose $\theta_{\text {vac }}^{(i)}=\theta_{\text {vac }}$ for $i=1,2$, and (13) can be written as

$$
\frac{d A_{\mathrm{geo}}}{d t}=-8 \pi \cdot \frac{M_{\mathrm{Pt}}}{\rho_{\mathrm{Pt}}} \cdot k_{1} \frac{u_{c}}{u^{\theta}} \cdot \theta_{\mathrm{vac}} \sum_{i=1,2} r_{i} N_{i}\left\{f_{1}\left(r_{i}\right)-\frac{\left[f_{1}\left(\bar{r}_{\mathrm{a}}\right)\right]^{2}}{f_{1}\left(r_{i}\right)}\right\}
$$

From the assumption that $\mathrm{Cpt}^{2+}$ is at steady state, we have

$$
\frac{d C_{\mathrm{Pt}^{2+}}}{d t}=0 \Rightarrow \sum_{i} r_{i}^{2} N_{i}\left\{f_{1}\left(r_{i}\right)-\frac{\left[f_{1}\left(\bar{r}_{\mathrm{a}}\right)\right]^{2}}{f_{1}\left(r_{i}\right)}\right\}=0
$$

Substitute the total surface area for the particles in the $i$-th group $a_{i}=4 \pi r_{i}^{2} N_{i} \quad(i=1,2)$, the above equation becomes

$$
\begin{gathered}
\sum_{i} r_{i}^{2} N_{i}\left\{f_{1}\left(r_{i}\right)-\frac{\left[f_{1}\left(\bar{r}_{\mathrm{a}}\right)\right]^{2}}{f_{1}\left(r_{i}\right)}\right\}=0 \Rightarrow \sum_{i} a_{i}\left\{f_{1}\left(r_{i}\right)-\frac{\left[f_{1}\left(\bar{r}_{\mathrm{a}}\right)\right]^{2}}{f_{1}\left(r_{i}\right)}\right\}=0 \\
\Rightarrow \frac{a_{1}}{a_{2}}=\frac{\frac{\left[f_{1}\left(\bar{r}_{\mathrm{a}}\right)\right]^{2}}{f_{1}\left(r_{2}\right)}-f_{1}\left(r_{2}\right)}{f_{1}\left(r_{1}\right)-\frac{\left[f_{1}\left(\bar{r}_{\mathrm{a}}\right)\right]^{2}}{f_{1}\left(r_{1}\right)}}=\frac{\frac{f_{1}\left(\bar{r}_{\mathrm{a}}\right)}{f_{1}\left(r_{2}\right)}-\frac{f_{1}\left(r_{2}\right)}{f_{1}\left(\bar{r}_{\mathrm{a}}\right)}}{\frac{f_{1}\left(r_{1}\right)}{f_{1}\left(\bar{r}_{\mathrm{a}}\right)}-\frac{f_{1}\left(\bar{r}_{\mathrm{a}}\right)}{f_{1}\left(r_{1}\right)}} \\
=\frac{\exp \left[\frac{F \alpha_{1}}{R T}\left(\frac{1}{\bar{r}_{\mathrm{a}}}-\frac{1}{r_{2}}\right)\right]-\exp \left[-\frac{F \alpha_{1}}{R T}\left(\frac{1}{\bar{r}_{\mathrm{a}}}-\frac{1}{r_{2}}\right)\right]}{\left.\left\lceil\frac{F \alpha_{1}}{R T}\left(\frac{1}{r_{1}}-\frac{1}{\bar{r}_{\mathrm{a}}}\right)\right]-\exp \left[-\frac{F \alpha_{1}}{R T}\left(\frac{1}{r_{1}}-\frac{1}{\bar{r}_{\mathrm{a}}}\right)\right]\right]} \Rightarrow \\
\left.\frac{\exp \left[\frac{F \alpha_{1}}{R T}\left(\frac{\Delta r_{2}}{\bar{r}_{\mathrm{a}} r_{2}}\right)\right]-\exp \left[-\frac{F \alpha_{1}}{R T}\left(\frac{\Delta r_{2}}{\bar{r}_{\mathrm{a}} r_{2}}\right)\right]}{\left[a_{2}\right.}=\frac{\exp \left[\frac{F \alpha_{1}}{R T}\left(\frac{\Delta r_{1}}{\bar{r}_{\mathrm{a}} r_{1}}\right)\right]-\exp \left[-\frac{F \alpha_{1}}{R T}\left(\frac{\Delta r_{1}}{\bar{r}_{\mathrm{a}} r_{1}}\right)\right]}{\text { (17) }}\right]
\end{gathered}
$$

when $\frac{\Delta r_{1}}{r_{1}}, \frac{\Delta r_{2}}{r_{2}}$ are small, $\frac{F \alpha_{1}}{R T}\left(\frac{\Delta r_{1}}{\bar{r}_{\mathrm{a}} r_{1}}\right), \frac{F \alpha_{1}}{R T}\left(\frac{\Delta r_{2}}{\overline{r_{\mathrm{a}}} r_{2}}\right)$ is also small, we can then make the following approximation by utilizing the Taylor expansion of the exponential function:

$$
\exp \left[\frac{F \alpha_{1}}{R T}\left(\frac{\Delta r_{i}}{\bar{r}_{\mathrm{a}} r_{i}}\right)\right]-\exp \left[-\frac{F \alpha_{1}}{R T}\left(\frac{\Delta r_{i}}{\overline{r_{\mathrm{a}}} r_{i}}\right)\right] \approx 2 \cdot \frac{F \alpha_{1}}{R T}\left(\frac{\Delta r_{i}}{\bar{r}_{\mathrm{a}} r_{i}}\right)
$$

Substitute (18) into (17), we have

$$
\frac{a_{1}}{a_{2}} \approx \frac{\Delta r_{2}}{r_{2}} / \frac{\Delta r_{1}}{r_{1}}
$$

Note that $a_{1}+a_{2}=A_{\text {geo }}$, then the total catalyst area in each group can be written as

$$
\left\{\begin{array}{l}
a_{1} \approx A_{\mathrm{geo}} \cdot \frac{\Delta r_{2}}{r_{2}} /\left(\frac{\Delta r_{1}}{r_{1}}+\frac{\Delta r_{2}}{r_{2}}\right) \\
a_{2} \approx A_{\mathrm{geo}} \cdot \frac{\Delta r_{1}}{r_{1}} /\left(\frac{\Delta r_{1}}{r_{1}}+\frac{\Delta r_{2}}{r_{2}}\right)
\end{array}\right.
$$

We already have from (15)

$$
\begin{aligned}
\frac{d A_{\mathrm{geo}}}{d t} & =-8 \pi \cdot \frac{M_{\mathrm{Pt}}}{\rho_{\mathrm{Pt}}} \cdot k_{1} \frac{u_{c}}{u^{\theta}} \cdot \theta_{\mathrm{vac}} \sum_{i=1,2} \frac{a_{i}}{4 \pi r_{i}} f_{1}\left(\bar{r}_{\mathrm{a}}\right)\left\{\frac{f_{1}\left(r_{i}\right)}{f_{1}\left(\bar{r}_{\mathrm{a}}\right)}-\frac{f_{1}\left(\bar{r}_{\mathrm{a}}\right)}{f_{1}\left(r_{i}\right)}\right\} \\
& =-8 \pi \cdot \frac{M_{\mathrm{Pt}}}{\rho_{\mathrm{Pt}}} \cdot k_{1} \frac{u_{c}}{u^{\theta}} \cdot \theta_{\mathrm{vac}} f_{1}\left(\bar{r}_{\mathrm{a}}\right) \frac{1}{4 \pi} \sum_{i=1,2} \frac{a_{i}}{r_{i}}\left\{\frac{f_{1}\left(r_{i}\right)}{f_{1}\left(\bar{r}_{\mathrm{a}}\right)}-\frac{f_{1}\left(\bar{r}_{\mathrm{a}}\right)}{f_{1}\left(r_{i}\right)}\right\}
\end{aligned}
$$

By substituting (20), the following approximation is obtained:

$$
\begin{aligned}
& \frac{d A_{\mathrm{geo}}}{d t} \approx-8 \pi \cdot \frac{M_{\mathrm{Pt}}}{\rho_{\mathrm{Pt}}} \cdot k_{1} \frac{u_{c}}{u^{\theta}} \cdot \theta_{\mathrm{vac}} f_{1}\left(\frac{3 \cdot V_{\mathrm{Pt}}}{A_{\mathrm{geo}}}\right) \cdot \frac{1}{4 \pi}\left\{\begin{array}{l}
\frac{a_{1}}{r_{1}}\left[2 \cdot \frac{F \alpha_{1}}{R T}\left(\frac{\Delta r_{1}}{\bar{r}_{\mathrm{a}}}\right)\right] \\
\left.-\frac{a_{2}}{r_{2}}\left[2 \cdot \frac{F \alpha_{1}}{R T}\left(\frac{\Delta r_{2}}{\bar{r}_{\mathrm{a}} r_{2}}\right)\right]\right\}
\end{array}\right\} \Rightarrow \\
& \frac{d A_{\mathrm{geo}}}{d t}=-4 k_{1} \cdot \frac{u_{c}}{u^{\theta}} \cdot \theta_{\mathrm{vac}} \cdot \frac{M_{\mathrm{Pt}}}{\rho_{\mathrm{Pt}}} \cdot \frac{F \alpha_{1}}{R T} \\
& \cdot f_{1}\left(\frac{3 \cdot V_{\mathrm{Pt}}}{A_{\mathrm{geo}}}\right) \cdot \frac{A_{\mathrm{geo}}}{3 V_{\mathrm{Pt}}}\left\{\frac{a_{1}}{r_{1}}\left(\frac{\Delta r_{1}}{r_{1}}\right)-\frac{a_{2}}{r_{2}}\left(\frac{\Delta r_{2}}{r_{2}}\right)\right\}
\end{aligned}
$$

Define $\frac{\Delta r_{2}}{\bar{r}_{\mathrm{a}}}=\beta_{r}, \Delta r_{1}=\alpha_{r} \Delta r_{2}$, the simplified aging model can finally be expressed as

$$
\left\{\begin{aligned}
\frac{d A_{g e o}}{d t}= & -4 k_{1} \cdot \frac{u_{c}}{u^{\theta}} \cdot \theta_{\mathrm{vac}} \cdot \frac{M_{\mathrm{Pt}}}{\rho_{\mathrm{Pt}}} \cdot \frac{F \alpha_{1}}{R T} \cdot \exp \left(\frac{F \alpha_{1}}{R T} \frac{A_{\mathrm{geo}}}{3 V_{\mathrm{Pt}}}\right) \\
& \cdot \frac{A_{\mathrm{geo}}^{3}}{9 V_{\mathrm{Pt}}^{2}} \frac{\alpha_{r} \beta_{r}^{2}}{\left(1+\beta_{r}\right)\left(1-\alpha_{r} \beta_{r}\right)} \\
\frac{d \theta_{\mathrm{PtO}}}{d t}= & \frac{v_{2}}{\Gamma_{\max }}-\frac{2 \theta_{\mathrm{P}_{\mathrm{P}}}}{\bar{r}_{\mathrm{a}}} \frac{d \bar{r}_{\mathrm{a}}}{d t}
\end{aligned}\right.
$$

where $\theta_{\text {vac }}=\max \left(0,1-\theta_{\mathrm{PtO}}\right)$. The degradation model is now simplified to a second-order system with the catalyst surface area $\left(A_{\text {geo }}\right)$ and the PtO coverage as the state variables. Also, in terms of factorability, the expression for degradation rate of $A_{\text {geo }}$ is in good shape since it can be factorized in the form of a product of a function of the current amount of damage $g_{2}\left(A_{\text {geo }}\right)$ times a function of the excitation amplitude $\mathrm{g}_{1}\left(\Delta \phi_{c}\right)$, as shown in (23)

$$
\frac{d A_{\text {geo }}}{d t}=\varepsilon \mathrm{g}_{1}\left(\Delta \phi_{c}\right) \cdot \mathrm{g}_{2}\left(A_{\mathrm{geo}}\right)
$$


The advantage of the degradation rate expression meeting condition (23) is that the Palmgren-Miner rule can now be tied to the damage accumulation equation. Basically, the Palmgren-Miner rule (Todinov, 2001) is an "additivity" property stating that it is possible to approximate the damage development under variable excitation amplitude by considering steps of constant amplitude and by summing the aging effects. This approach is used, for example, in [23], [24] to track damage evolution in machinery.

Simulations are carried out for the model validation by comparing the simple model output with the detailed one. The results are shown below in Figure 10 and Figure 11. Respectively, a constant $0.97 \mathrm{~V}$ and a cycling potential profile from $0.8 \mathrm{~V} \sim 0.97 \mathrm{~V}$ are used as the input potential loads to emulate the OCV aging condition and a working fuel cell operating condition. In both cases, the model parameters are chosen to be $\alpha_{r}=1.1$, and $\beta_{r}=0.038$. It can be seen that, by tuning the model parameters, the simple model achieves good approximation to the more complex one. The right plots of the figures show the evolution of the average radius $\bar{r}_{\mathrm{a}}$ and the two fictitious radii $r_{1}$ and $r_{2}$.
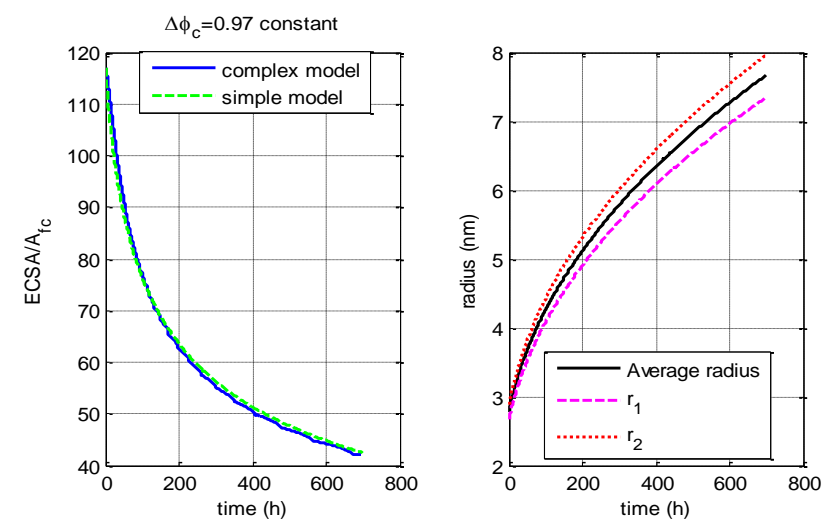

Figure 10. Comparison between the catalyst magnifying coefficient evolution for 64-particle model (constant $0.97 \mathrm{~V}$ )
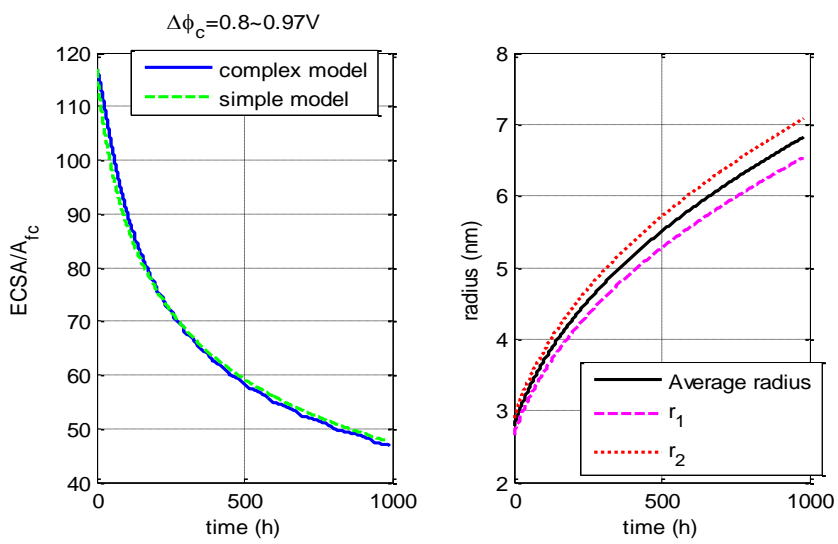

Figure 11 Model comparison (cycling potential $0.8 \mathrm{~V}$ $0.97 \mathrm{~V})$

\section{A UKF-BASED FRAMEWORK}

\subsection{Bayesian framework for joint estimation}

We first consider a general joint estimation problem based on the following discrete system

$$
\begin{gathered}
\mathbf{x}_{k+1}=\mathbf{f}\left(\mathbf{x}_{k}, \mathbf{u}_{k}, \mathbf{v}_{k}, \mathbf{w}_{k}\right) \\
\mathbf{y}_{k}=\mathbf{h}\left(\mathbf{x}_{k}, u_{k}, \mathbf{n}_{k}, w_{k}\right)
\end{gathered}
$$

where $\mathbf{x}_{\mathbf{k}}$ represent the states of the system, $\mathbf{y}_{\mathbf{k}}$ the outputs, $\mathbf{u}_{\mathbf{k}}$ the inputs $, \mathbf{v}_{\mathbf{k}}, \mathbf{n}_{\mathbf{k}}$ the process and measurement noises, respectively, and $\mathbf{w}_{\mathbf{k}}$ the time-varying system parameters. $\mathbf{f}$ is the state equation representing the system dynamics, which is generally nonlinear; and $\mathbf{h}$ is the measurement equation, in the form of a nonlinear map in general.

Since the parameters $\mathbf{w}_{\mathbf{k}}$ are unknown and time-varying, the state and parameter must be simultaneously and jointly estimated based on the noisy measured output. On the other hand, the dynamics of the time-varying system parameters are usually hard to describe, i.e., there is a lack of descriptive dynamic equation to characterize the parameters. A commonly used method to address this issue is to treat the parameter as a stochastic signal driven by a white noise $\left(\mathbf{r}_{\mathbf{k}}\right)$

$$
\mathbf{w}_{\mathrm{k}+1}=\mathrm{w}_{\mathrm{k}}+\mathrm{r}_{\mathrm{k}}
$$

Then, by concatenating the states and parameters to form an augmented state vector $\mathbf{x}_{\mathbf{k}}^{a}=\left[\begin{array}{ll}\mathbf{x}_{\mathbf{k}}^{T} & \mathbf{w}_{\mathbf{k}}^{T}\end{array}\right]^{T}$, joint state space equations (Wan \& Merwe, n.d.) (assuming additive noises) can be obtained as follows

$$
\begin{aligned}
\mathbf{x}_{\mathbf{k}+1}^{a} & =\left[\begin{array}{l}
\mathbf{x}_{\mathbf{k}+1} \\
\mathbf{w}_{\mathbf{k}+1}
\end{array}\right]=\left[\begin{array}{c}
\mathbf{f}\left(\mathbf{x}_{\mathbf{k}}, \mathbf{u}_{\mathbf{k}}, \mathbf{w}_{\mathbf{k}}\right) \\
\mathbf{I} \cdot \mathbf{w}_{\mathbf{k}}
\end{array}\right]+\left[\begin{array}{c}
\mathbf{B} \cdot \mathbf{v}_{\mathbf{k}} \\
\mathbf{r}_{\mathbf{k}}
\end{array}\right] \\
& =\mathbf{F}\left(\mathbf{x}_{\mathbf{k}}^{a}, \mathbf{u}_{\mathbf{k}}\right)+\left[\begin{array}{c}
\mathbf{B} \cdot \mathbf{v}_{\mathbf{k}} \\
\mathbf{r}_{\mathbf{k}}
\end{array}\right] \\
\mathbf{y}_{\mathbf{k}} & =\mathbf{h}\left(\mathbf{x}_{\mathbf{k}}, \mathbf{u}_{\mathbf{k}}, \mathbf{w}_{\mathbf{k}}\right)+\mathbf{n}_{\mathbf{k}}=\mathbf{H}\left(\mathbf{x}_{\mathbf{k}}^{a}, \mathbf{u}_{\mathbf{k}}\right)+\mathbf{n}_{\mathbf{k}}
\end{aligned}
$$

The joint estimation problem of states and parameters based on observation can be formulated in an optimal recursive estimation framework as given in the following equation

$$
\hat{\mathbf{x}}_{\mathbf{k}}^{a}=E\left[\mathbf{x}_{\mathbf{k}}^{a} \mid \mathbf{Y}_{\mathbf{k}}\right]=E\left[\mathbf{x}_{\mathbf{k}}^{a} \mid \mathbf{y}_{\mathbf{0}}, \mathbf{y}_{\mathbf{1}}, \cdots \mathbf{y}_{\mathbf{k}}\right]
$$

Two step process (recursively) are involved, the first is the measurement correction

$$
p\left(\mathbf{x}_{\mathbf{k}}^{a} \mid \mathbf{Y}_{\mathbf{k}}\right)=\frac{p\left(\mathbf{x}_{\mathbf{k}}^{a} \mid \mathbf{Y}_{\mathbf{k}-1}\right) p\left(\mathbf{y}_{\mathbf{k}} \mid \mathbf{x}_{\mathbf{k}}^{a}\right)}{p\left(\mathbf{y}_{\mathbf{k}} \mid \mathbf{Y}_{\mathbf{k}-1}\right)}
$$

where $p($.$) indicates the probability density function.$ 
And the second is the one-step prediction

$$
p\left(\mathbf{x}_{\mathbf{k}+1}^{a} \mid \mathbf{Y}_{\mathbf{k}}\right)=\int p\left(\mathbf{x}_{\mathbf{k}+1}^{a} \mid \mathbf{x}_{\mathbf{k}}^{a}\right) p\left(\mathbf{x}_{\mathbf{k}}^{a} \mid \mathbf{Y}_{\mathbf{k}}\right) d \mathbf{x}_{\mathbf{k}}^{a}
$$

Various filtering techniques can be implemented in this general recursive estimation framework, including the most widely used extended Kalman filter (EKF), particle filtering (PF), and unscented Kalman filter (UKF). EKF is difficult to tune, and the Jacobian is usually hard to derive, and it can only handle limited amount of nonlinearity; while PF can handle arbitrary distributions and nonlinearities but is computationally very complex. In this paper, we focus on the UKF approach since we believe it gives a nice tradeoff between PF and EKF.

\subsection{UKF implementation}

We assume the additive (zero mean) noise case and follow the UKF procedure given in (Wan \& Merwe, n.d.).

First, the augmented state estimation and covariance matrix are initialized with (32) and (33).

$$
\begin{gathered}
\hat{\mathbf{x}}_{0}^{a}=E\left[\mathbf{x}_{0}^{a}\right] \\
\mathbf{P}_{0}^{a}=E\left[\left(\mathbf{x}_{0}^{a}-\hat{\mathbf{x}}_{0}^{a}\right)\left(\mathbf{x}_{0}^{a}-\hat{\mathbf{x}}_{0}^{a}\right)^{T}\right]
\end{gathered}
$$

Then, for each iteration $(k=1,2, \ldots)$, the sigma points for the state variables in the last step are obtained and concatenated to form a matrix as follows

$$
\mathbf{X}_{k-1}=\left[\begin{array}{lll}
\hat{\mathbf{x}}_{k-1}^{a} & \hat{\mathbf{x}}_{k-1}^{a}+c \sqrt{\mathbf{P}_{k-1}^{a}} & \hat{\mathbf{x}}_{k-1}^{a}-c \sqrt{\mathbf{P}_{k-1}^{a}}
\end{array}\right]
$$

where $\lambda=\alpha^{2}(L+\kappa)-L, c=L+\lambda, L$ is the dimension of the system given by (27), and $\alpha, \kappa$ and $\beta$ are all tunable parameters (in this paper, $\alpha=10^{-3}, \kappa=0, \beta=2$ is chosen according to (Wan \& Merwe, n.d.). Note that the addition (or subtraction) in (34) is not a strict mathematical notation, but rather a concise notation adopted from m-script of Matlab that actually adds (or subtracts) a vector to every column of a matrix.

These sigma points are then fed to the state equation to generate a new set of sigma points for the state variables in the current step: $\mathbf{X}_{k \mid k-1}^{(i)}=\mathbf{F}\left(\mathbf{X}_{k-1}^{(i)}, \mathbf{u}_{\mathbf{k}}\right)$, where the superscript (i) denotes the $i$-th column of the corresponding matrix, i.e., the $i$-th sigma point, and $\mathbf{F}$ as in (27).

The one-step prediction for the augmented state vector in (31), when implemented with UKF approach, can now be expressed as

$$
\hat{\mathbf{x}}_{k}^{a-}=\sum_{i=0}^{2 L} w_{i}^{m} \cdot \mathbf{X}_{k \mid k-1}^{(i)}
$$

$$
\mathbf{P}_{k}^{a-}=\sum_{i=0}^{2 L} w_{i}^{c} \cdot\left[\mathbf{X}_{k \mid k-1}^{(i)}-\hat{\mathbf{x}}_{k}^{a-}\right]\left[\mathbf{X}_{k \mid k-1}^{(i)}-\hat{\mathbf{x}}_{k}^{a-}\right]^{T}+\mathbf{Q}
$$

where $\mathbf{Q}$ is the process noise covariance matrix, and $w_{i}^{m}, w_{i}^{c}$ are the weights for the corresponding sigma points.

Measurement correction, on the other hand, is given through (37) (43)

$$
\begin{gathered}
\mathbf{Y}_{k \mid k-1}^{(i)}=\mathbf{H}\left(\mathbf{X}_{k \mid k-1}^{(i)}, \mathbf{u}_{\mathbf{k}}\right) \\
\hat{\mathbf{y}}_{k}^{-}=\sum_{i=0}^{2 L} w_{i}^{\mathrm{m}} \cdot \mathbf{Y}_{k \mid k-1}^{(i)} \\
\mathbf{P}_{\overline{\mathbf{y}}_{k} \bar{y}_{k}}=\sum_{i=0}^{2 L} w_{i}^{\mathrm{c}} \cdot\left[\mathbf{Y}_{k \mid k-1}^{(i)}-\hat{\mathbf{y}}_{k}^{-}\right]\left[\mathbf{Y}_{k \mid k-1}^{(i)}-\hat{\mathbf{y}}_{k}^{-}\right]^{T}+\mathbf{R} \\
\mathbf{P}_{\overline{\mathbf{x}}_{k} \overline{\mathbf{y}}_{k}}=\sum_{i=0}^{2 L} w_{i}^{\mathrm{c}} \cdot\left[\mathbf{X}_{k \mid k-1}^{(i)}-\hat{\mathbf{x}}_{k}^{a-}\right]\left[\mathbf{Y}_{k \mid k-1}^{(i)}-\hat{\mathbf{y}}_{k}^{-}\right]^{T} \\
\mathbf{K}_{k}=\mathbf{P}_{\overline{\mathbf{x}}_{k} \overline{\mathbf{y}}_{k}} \mathbf{P}_{\overline{\mathbf{y}}_{k} \overline{\mathbf{y}}_{k}}^{-1} \\
\hat{\mathbf{x}}_{k}^{a}=\hat{\mathbf{x}}_{k}^{a-}+\mathbf{K}_{k}\left(\mathbf{y}_{k}-\hat{\mathbf{y}}_{k}^{-}\right) \\
\mathbf{P}_{k}^{a}=\mathbf{P}_{k}^{a-}-\mathbf{K}_{k} \mathbf{P}_{\overline{\mathbf{y}}_{k} \bar{y}_{k}} \mathbf{K}_{k}^{T}
\end{gathered}
$$

where $\mathbf{R}$ is the measurement noise covariance matrix, and $\mathbf{H}$ as in (27).

The weights typically employed in UKF are given as follows

$w_{0}^{m}=\lambda / c, w_{0}^{m}=\lambda / c+\left(1-\alpha^{2}+\beta\right), w_{i}^{m}=w_{i}^{c}=\lambda / 2 c$.

\subsection{UKF Approach for Prediction}

The Bayesian method is an iterative method that involves two steps in each iteration, i.e., prediction and measurement correction. In the general Bayesian estimation framework, the one-step prediction in (31) can be extended to $(m+1)$ step long-term prediction as follows,

$$
\begin{aligned}
p\left(\mathbf{x}_{k+m+1}^{a} \mid \mathbf{Y}_{k}\right) & =\int p\left(\mathbf{x}_{k+m+1}^{a} \mid \mathbf{x}_{k+m}^{a}\right) p\left(\mathbf{x}_{k+m}^{a} \mid \mathbf{Y}_{k}\right) d \mathbf{x}_{k+m}^{a} \\
& =\int p\left(\mathbf{x}_{k+m+1}^{a} \mid \mathbf{x}_{\mathbf{k}+m}^{a}\right)\left[\int p\left(\mathbf{x}_{k+m}^{a} \mid \mathbf{x}_{k+m-1}^{a}\right) p\left(\mathbf{x}_{k+m-1}^{a} \mid \mathbf{Y}_{k}\right) d \mathbf{x}_{k+m-1}^{a}\right] d \mathbf{x}_{k+m}^{a} \\
& =\iint p\left(\mathbf{x}_{k+m+1}^{a} \mid \mathbf{x}_{k+m}^{a}\right) p\left(\mathbf{x}_{k+m}^{a} \mid \mathbf{x}_{k+m-1}^{a}\right) p\left(\mathbf{x}_{k+m-1}^{a} \mid \mathbf{Y}_{k}\right) d \mathbf{x}_{k+m-1}^{a} d \mathbf{x}_{k+m}^{a} \\
& =\int \cdots \int p\left(\mathbf{x}_{k}^{a} \mid \mathbf{Y}_{k}\right) \prod_{j=k}^{k+m} p\left(\mathbf{x}_{j+1}^{a} \mid \mathbf{x}_{j}^{a}\right) d \mathbf{x}_{k}^{a} \cdots d \mathbf{x}_{k+m}^{a}
\end{aligned}
$$

In this case of $(m+1)$-step long-term prediction, when implemented with UKF approach, the equations (35) can be written as (45), 


$$
\begin{aligned}
& \mathbf{X}_{k+m+1 \mid k}^{(i)}=\mathbf{F}\left(\mathbf{X}_{k+m \mid k}^{(i)}, \mathbf{u}_{k+m+1}\right) \\
& \hat{\mathbf{x}}_{k+m+1 \mid k}^{a}=\sum_{i=0}^{2 L} w_{i}^{\mathrm{m}} \cdot \mathbf{X}_{k+m+1 \mid k}^{(i)} \\
& \mathbf{P}_{k+m+1 \mid k}^{a}=\sum_{i=0}^{2 L} w_{i}^{\mathrm{c}} \cdot\left[\mathbf{X}_{k+m+1 \mid k}^{(i)}-\hat{\mathbf{x}}_{k+m+1 \mid k}^{a}\right]\left[\mathbf{X}_{k+m+1 \mid k}^{(i)}-\hat{\mathbf{x}}_{k+m+1 \mid k}^{a}\right]^{T}+\mathbf{Q}
\end{aligned}
$$

As can be seen from (45), the long-term prediction utilizes only the one-step prediction iteratively without measurement correction, since the future measurement is unavailable at the current step when prediction is made. Equivalently, the standard UKF procedure can be used with the Kalman gain in (42) set to 0, i.e., $\mathbf{K}_{k}=\mathbf{0}$.

Also note that in (45), the system input $\mathbf{u}_{n}$ at step $n(n>k)$ is required to generate the new set of sigma points at each iteration. Generally speaking, the future input can be obtained by analyzing its past stochastic feature and then projecting into the future, while at the same time including the uncertainty of the input itself. For simplicity purpose, in this paper, the current input $\mathbf{u}_{k}$ is taken as the constant input for all future steps during the prediction, and the uncertainty issue is not considered.

\section{Application Of the Aging MOdel for PHM}

\subsection{Damage Tracking and Prognostics for Catalyst Degradation}

As a first step, we investigate the health monitoring and prognostic problem for the catalyst degradation only.

In Section 2, we have already established a prognosticoriented fuel cell aging model to describe the catalyst degradation process. The model is a second order system with simple structure. For simplicity of demonstration, we further simplify that model to a first order dynamic model, as shown in (46), by neglecting the dynamics of the platinum oxide coverage during the load cycling.

$$
\frac{d A_{\mathrm{geo}}}{d t} \approx-\frac{4 k_{1}}{9 V_{\mathrm{Pt}}^{2}} \cdot \frac{u_{c}}{u^{\theta}} \cdot \theta_{\mathrm{vac}} \cdot \frac{M_{\mathrm{Pt}}}{\rho_{\mathrm{Pt}}} \cdot \frac{F \alpha_{1}}{R T} \cdot \exp \left(\frac{F \alpha_{1}}{R T} \frac{A_{\mathrm{geo}}}{3 V_{\mathrm{Pt}}}\right) \cdot A_{\mathrm{geo}}^{3} \alpha_{r} \beta_{r}^{2}
$$

Since $k_{1}$ is extremely small in value, we can scale the above equation in time with a scaling coefficient $\varepsilon$ to denote its slowness explicitly. Also note that $k_{1}$ is an implicit function of the temperature $T$, thus we can write the degradation rate of the catalytic surface area as a function of the input potential load, the temperature, the parameter $\beta_{r}$, and the catalytic surface area itself.

$$
\frac{d A_{\mathrm{geo}}}{d t}=\varepsilon \cdot \mathbf{g}\left(A_{\mathrm{geo}}, \Delta \phi_{c}, \beta_{r}, T\right)
$$

As we mentioned above, dynamics of the platinum oxide coverage is neglected in this first order system. Thus the platinum oxide coverage is considered as a static function of the equivalent load input: $\theta_{\text {vac }}=\operatorname{map}\left(u_{c}\right)$. Now, by taking into account the uncertain factors as additive process noise, the following stochastic degradation model can be obtained

$$
\begin{aligned}
\frac{d A_{\mathrm{geo}}}{d t} & =\varepsilon \cdot \mathbf{g}\left(A_{\mathrm{geo}}, \Delta \phi_{c}, \beta_{r}, T\right)+w_{1}(t) \\
\dot{\beta}_{r} & =w_{2}(t)
\end{aligned}
$$

where the unknown time-varying parameter $\beta_{r}$ is taken as a state variable, and its derivative as a process noise.

The cell voltage given in (49) is utilized as the measured system output,

$$
\begin{gathered}
V_{\text {cell }}=E_{0}\left(T_{\mathrm{fc}}\right)-\frac{R T_{\mathrm{fc}}}{4(1-\alpha) F} \ln \left(i+i_{\text {leak }}\right)+\frac{R T_{\mathrm{fc}}}{4(1-\alpha) F} \ln \xi_{\mathrm{cat}} \\
+\frac{R T_{\mathrm{fc}}}{2 F} \ln p_{H_{2}}^{*}+\frac{R T_{\mathrm{fc}}}{4(1-\alpha) F} \ln p_{O_{2}}^{*}-i \cdot A_{\mathrm{fc}} \cdot R_{\text {ohm }}
\end{gathered}
$$

where $E_{0}\left(T_{\mathrm{fc}}\right)$ is the voltage component that only depends on the fuel cell temperature for a specific type of fuel cell; $\alpha$ is the transfer coefficient of the oxygen reduction reaction; $i$ and $i_{\text {leak }}$ are the current density and the leak current density; $p_{\mathrm{H}_{2}}^{*}, p_{\mathrm{O}_{2}}^{*}$ are the hydrogen and oxygen partial pressures at the reaction site; and $R_{\mathrm{ohm}}$ is the total ohmic resistance of the fuel cell. $\xi_{\text {cat }}=\frac{A_{\text {geo }}}{\left.A_{\text {geo }}\right|_{t=0}}$ is the ratio of current and initial catalytic geometric (or equivalently electrochemical) surface area, the value of which is 1 when the fuel cell is at its fresh state.

From (49), it is obvious that the measured voltage is not only a function of the slowly varying damage variables, i.e, $i_{\text {leak }}$ and $\xi_{\text {cat }}$, but also a function of the fast varying system variables $p_{\mathrm{H}_{2}}^{*}, p_{\mathrm{O}_{2}}^{*}$. To obtain an output variable that is solely related to the slowly varying damage variables, i.e., the state variables in the aging model describing the degradation process, we propose an output acquisition system as shown in Figure 12.

Basically, the output acquisition method utilizes an agingfree dynamic model of the fuel cell system that captures the fast dynamics of the FCS in the normal time scale while assuming constant, non-degrading damage variables in the long time scale. The model output is given in (50).

$$
\begin{gathered}
\hat{V}_{\text {cell }}=E_{0}\left(T_{\mathrm{fc}}\right)+\frac{R T_{\mathrm{fc}}}{2 F} \ln \hat{p}_{\mathrm{H}_{2}}^{*}+\frac{R T_{\mathrm{fc}}}{4(1-\alpha) F} \ln \hat{p}_{O_{2}}^{*} \\
-i \cdot A_{\mathrm{fc}} \cdot R_{\text {ohm }}-\frac{R T_{\mathrm{fc}}}{4(1-\alpha) F} \ln \left(i+i_{\text {leak }}^{0}\right)
\end{gathered}
$$


where $i_{\text {leak }}^{0}$ is the membrane leak current density at the initial time, and here we assume that the total ohmic resistance $R_{\text {ohm }}$ is readily available.

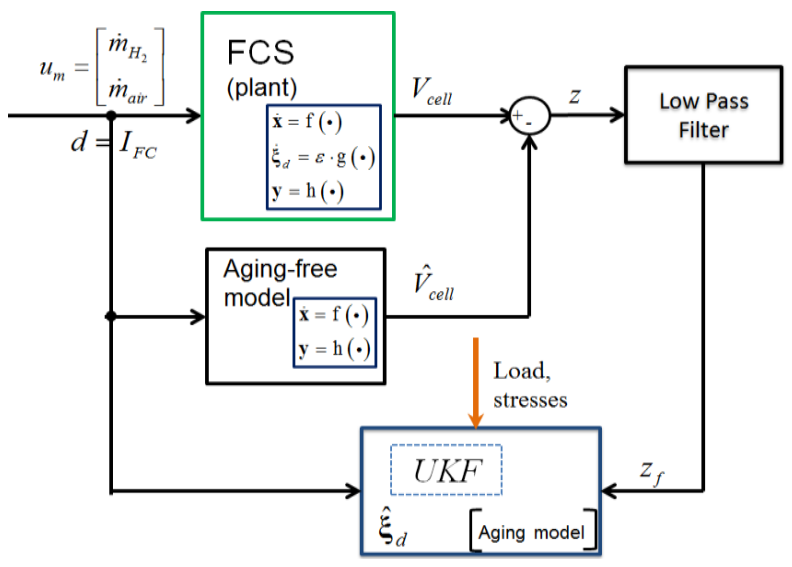

Figure 12 Acquisition of the output signal for UKF

The difference of the outputs of the actual FCS and the aging-free dynamic FCS model can then be calculated and written as follows.

$$
\begin{aligned}
V_{\text {cell }}-\hat{V}_{\text {cell }}= & \frac{R T_{\mathrm{fc}}}{4(1-\alpha) F} \ln \xi_{\text {cat }}-\frac{R T_{\mathrm{fc}}}{4(1-\alpha) F} \ln \left(\frac{i+i_{\text {leak }}}{i+i_{\text {leak }}^{0}}\right) \\
& +\left(\frac{R T_{\mathrm{fc}}}{2 F} \ln \frac{p_{\mathrm{H}_{2}}^{*}}{\hat{p}_{\mathrm{H}_{2}}^{*}}\right)+\left\lceil\frac{R T_{\mathrm{fc}}}{4(1-\alpha) F} \ln \frac{p_{\mathrm{O}_{2}}^{*}}{\hat{p}_{\mathrm{O}_{2}}^{*}}\right\rceil
\end{aligned}
$$

Ideally, given that the model is accurate in capturing the fast dynamics of the system and no disturbance is present, we have $\frac{p_{H_{2}}^{*}}{\hat{p}_{H_{2}}^{*}}=1, \frac{p_{O_{2}}^{*}}{\hat{p}_{O_{2}}^{*}}=1$, and the difference in (51) would depend only on the slowly varying damage variables. However, in practice, the instantaneous voltage degradation $\left(V_{\text {cell }}-\hat{V}_{\text {cell }}\right)$ would inevitably be affected by modeling error that may result in model prediction deviation especially in transition, and by disturbances such as the water content in the gas diffusion media that could even result in offset between the two outputs at their steady states. By denoting the two kinds of sources of mismatches with $n_{d}$ and $n_{s}$ respectively, the difference $z$ in Figure 12 can be expressed as follows,

$$
z=\frac{R T_{\mathrm{fc}}}{4(1-\alpha) F} \ln \xi_{\text {cat }}-\frac{R T_{\mathrm{fc}}}{4(1-\alpha) F} \ln \left(\frac{i+i_{\text {leak }}}{i+i_{\text {leak }}^{0}}\right)+n_{d}+n_{s}
$$

The deviation between the model and the actual plant outputs is then fed to the low pass filter in Figure 12 to remove the fast dynamics mismatch $n_{d}$ and to obtain the filtered deviation $z_{f}$ as in (53).

$$
z_{f}=\frac{R T_{\mathrm{fc}}}{4(1-\alpha) F} \ln \xi_{\mathrm{cat}}-\frac{R T_{\mathrm{fc}}}{4(1-\alpha) F} \ln \left(\frac{i+i_{\text {leak }}}{i+i_{\text {leak }}^{0}}\right)+\bar{n}_{s}
$$

where $\bar{n}_{s}$ contains also the distortion effects introduced by the filter on $z$.

To apply the UKF approach introduced in the previous section, we need to first discretize the continuous system equations. By denoting the fixed sample time as $\Delta T$, the state equations can be written as

$$
\begin{aligned}
A_{\mathrm{geo}, k+1} & =\varepsilon \cdot N_{\varepsilon} \cdot \Delta T \cdot \mathbf{g}\left(A_{\mathrm{geo}, k}, \Delta \phi_{c, k}, \beta_{r, k}, T_{\mathrm{fc}}\right)+\varepsilon \cdot N_{\varepsilon} \cdot \Delta T \cdot w_{1, k} \\
\beta_{r, k+1} & =\beta_{r, k}+\varepsilon \cdot N_{\varepsilon} \cdot \Delta T \cdot w_{2, k}
\end{aligned}
$$

and the output equation is given by (55)

$$
z_{f, k}=\frac{R T_{\mathrm{fc}}}{4(1-\alpha) F} \ln \xi_{\mathrm{cat}, k}-\frac{R T_{\mathrm{fc}}}{4(1-\alpha) F} \ln \left(\frac{i_{k}+i_{\text {leak }, k}}{i_{k}+i_{\text {leak }, k}^{0}}\right)+\bar{n}_{s, k}
$$

where the $k$ represents the discretization time step. Note that, since the fuel cell catalyst degradation is an extremely slow process (the fresh to failure life cycle typically takes several hundred hours or longer), the sample time $\Delta T$ for the discretized model employed by the UKF can be chosen in the hour scale, instead of second, to lower the unnecessary computational burden. In this paper, we choose $\Delta T=1500 \mathrm{~s}$ (25 min). Under such a slow time scale, disturbances induced offset between the two voltage outputs $\bar{n}_{s, k}$ can then be treated reasonably as a white noise.

As seen in Figure 12, $z_{f, k}$ in (55) can be practically obtained as

$$
z_{f, k}=\operatorname{LPF}\left(V_{\text {cell }, k}-\hat{V}_{\text {cell }, k}\right)
$$

where LPF stands for the low pass filter.

Simulation is carried out where we assume a cyclic load profile as given in Figure 13.
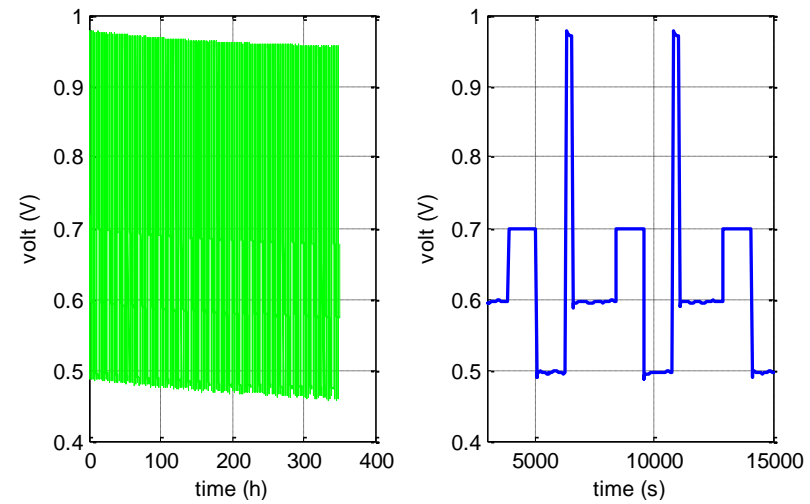

Figure 13 Load profile for PHM scheme simulation validation 
The simulation result for health monitoring validation is presented in Figure 14, where the estimated damage variable is compared with the "real" value obtained from the 64particle catalyst aging model. It can be seen that the ECSA has been tracked satisfactorily, while $\beta_{r}$ varies between the range [0.03, 0.04], and gradually settles down after 100 hours. The result also justifies our previous assumption that $\frac{\Delta r_{1}}{\overline{r_{\mathrm{a}}}}$ and $\frac{\Delta r_{2}}{\bar{r}_{\mathrm{a}}}$ are constant during the aging process. In fact, the first 100 hours can be considered as the necessary phase for parameter identification. After this phase, the parameter $\beta_{r}$ would stay relatively stable around some constant value. We will see later that this characteristic feature can be exploited to do fault diagnostics for the purpose of early detection of the massive gas crossover.

The parameters used for this simulation are grouped in the following table:

\section{Table 1 Parameters used in the simulation}

\begin{tabular}{|c|c|c|c|}
\hline \multirow{2}{*}{$\begin{array}{l}\text { Initializa- } \\
\text { tion }\end{array}$} & $\begin{array}{l}\text { Initial } \\
\text { state } \\
\text { estimate }\end{array}$ & {$\left[\begin{array}{c}\hat{A}_{P t}^{0} \\
\hat{\beta}_{r}^{0}\end{array}\right]=\left[\begin{array}{c}A_{P t}^{0} \times 1.1 \\
0.035\end{array}\right]$} & \\
\hline & $\begin{array}{l}\text { Initial } \\
\text { covarian } \\
\text { ce matrix }\end{array}$ & $P_{0}=\left[\begin{array}{c}\left(0.02 \times A_{P t}^{0}\right)^{2} \\
0\end{array}\right.$ & $\begin{array}{c}0 \\
(0.04 \times 0.15)^{2}\end{array}$ \\
\hline \multirow{2}{*}{$\begin{array}{l}\text { Noise } \\
\text { covariance } \\
\text { matrix }\end{array}$} & $\begin{array}{l}\text { Process } \\
\text { noise }\end{array}$ & {$\left[\begin{array}{ll}Q_{1} & 0 \\
0 & Q_{2}\end{array}\right]=\left[\begin{array}{c}\left(A_{P_{t}}^{0} \times 2 \times 10^{-6}\right)^{2} \\
0\end{array}\right.$} & $\begin{array}{c}0 \\
\left(0.035 \times 2 \times 10^{-6}\right)^{2}\end{array}$ \\
\hline & $\begin{array}{l}\text { Measure- } \\
\text { ment } \\
\text { noise }\end{array}$ & $R=6.7 \times 10^{-6}$ & \\
\hline
\end{tabular}
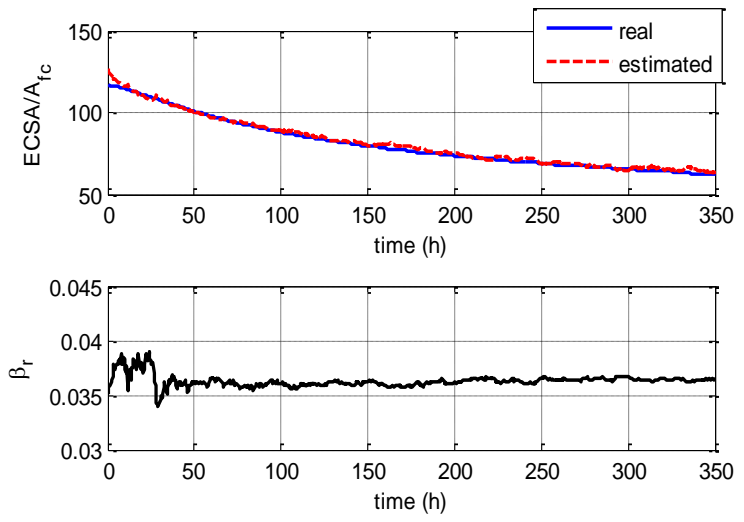

Figure 14 Simulation result for health monitoring

The simulation results for prognostic validation are presented in Figure 15 and Figure 16.

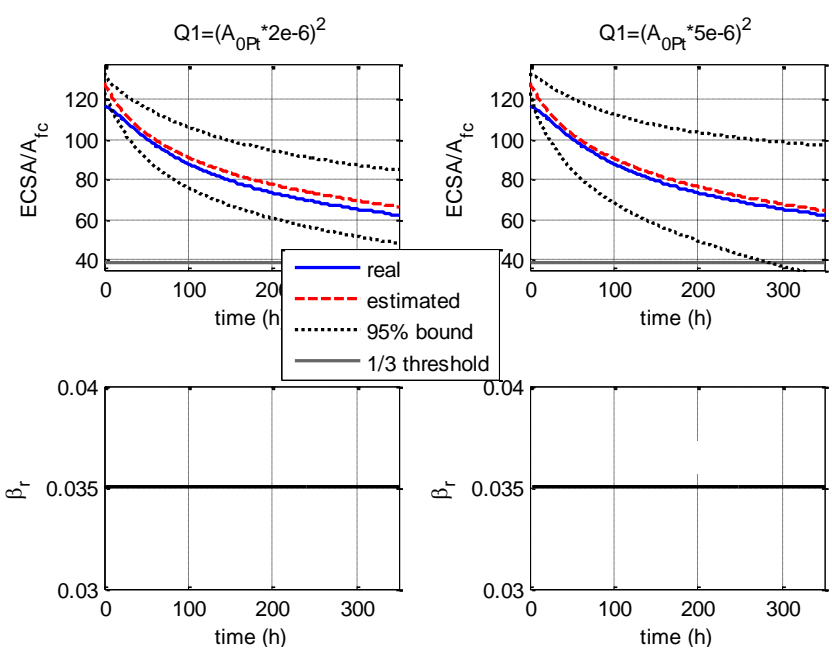

Figure 15 Simulation result for prognostics at the beginning of life

In Figure 15, prognostic is performed at the very beginning of life of the fuel cell. It can be seen that the $95 \%$ confidence interval is growing larger with increasing prediction step due to lack of measurement correction. $\beta_{r}$ is seen to be constant all the time with the same reason. Two values for the process noise $Q_{1}$ are compared, i.e., $\left(A_{P t}^{0} \times 2 \times 10^{-6}\right)^{2}$ and $\left(A_{P t}^{0} \times 5 \times 10^{-6}\right)^{2}$. As expected, the smaller value of the process noise results in a narrower $95 \%$ bound, and thus a more accurate prediction.

Figure 16 shows the simulation results with prognostic starting from the middle of the durability test, here at 100 hours. Due to lack of measurement correction, $\beta_{r}$ stops updating and is taken as a constant after100 hours. The $95 \%$ confidence interval is also seen growing larger with increasing prediction step, but much narrower compared to that in Figure 15.

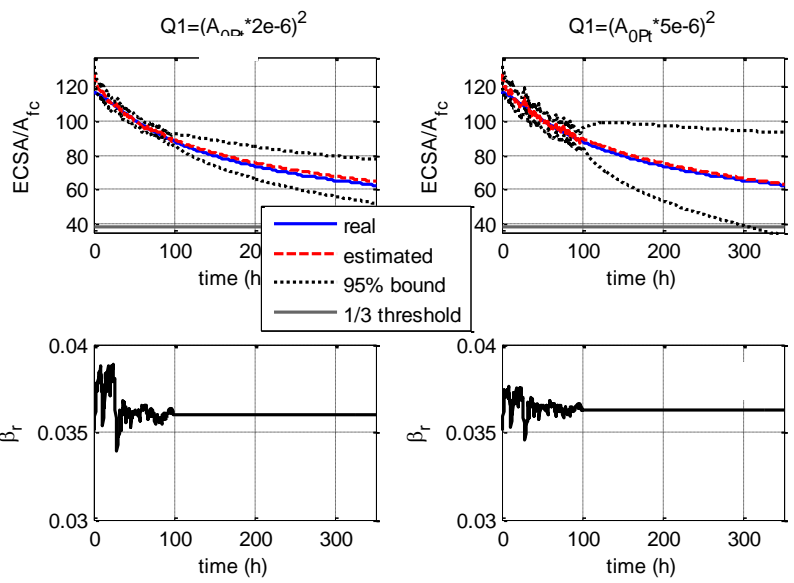

Figure 16 Simulation result for Prognostics at $100 \mathrm{~h}$ 


\subsection{Fault Detection of the Initiation of Massive Hydrogen Crossover}

The initiation of massive gas crossover through the membrane is rather stochastic and can be very difficult to predict. On the other hand, the time of the initiation is also very critical since it indicates the beginning of the end phase of the fuel cell lifetime: the massive gas crossover would accelerate exponentially after its initiation, resulting in a "quick death" of the fuel cell. Therefore, from a prognostic point of view, it is extremely important to be able to detect this initiation time at an early stage. A prognostic scheme for the membrane damage can then be activated after the fault detection to track the membrane health and predict the remaining useful life of the fuel cell. In this subsection, we utilize the health monitoring scheme developed for catalyst degradation to detect this incipient membrane failure.

We conduct the simulation study with the current load profile presented in Figure 17. Membrane crossover fault is injected at 250 hour to grow exponentially afterwards. The corresponding output voltage is presented in Figure 18. The gradual performance loss phase from $0 \sim 250 \mathrm{~h}$ and the sharp performance loss phase from $250 \mathrm{~h} \sim 350 \mathrm{~h}$ can be clearly seen in the figure. Measurement noise covariance matrix used in this simulation is $R=1 \times 10^{-4}$.

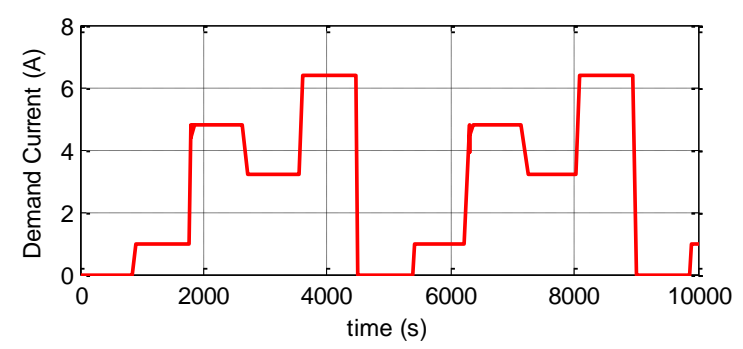

Figure 17 Current profile for the aging simulation
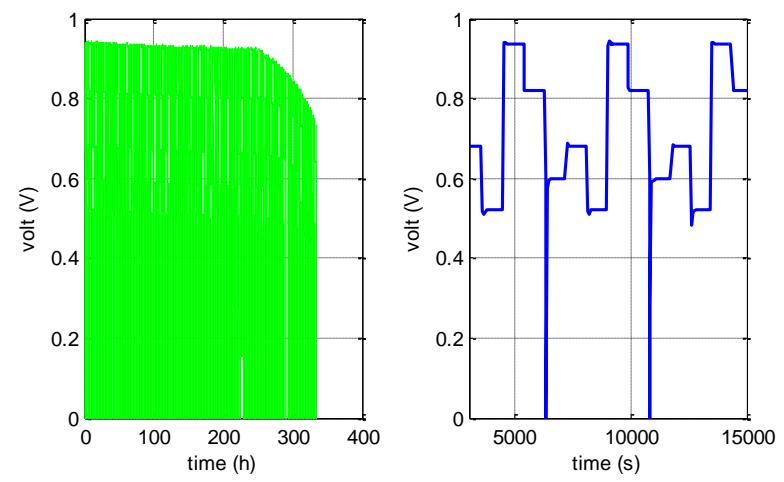

Figure 18 Voltage output from the simulated aging test

The simulation results for the damage tracking of ECSA and detection of current leak are shown in Figure 19. It can be seen that the parameter $\beta_{r}$ can be utilized as a good indicator for the early detection of the initiation of massive hydrogen crossover. By appropriately choosing the threshold, the serious current leak can be detected shortly after its initiation, in this case, less than $30 \mathrm{~h}$. Note that the aging model information of the membrane, i.e., the dynamics for the leak current density growth is not necessary for the application of this scheme.
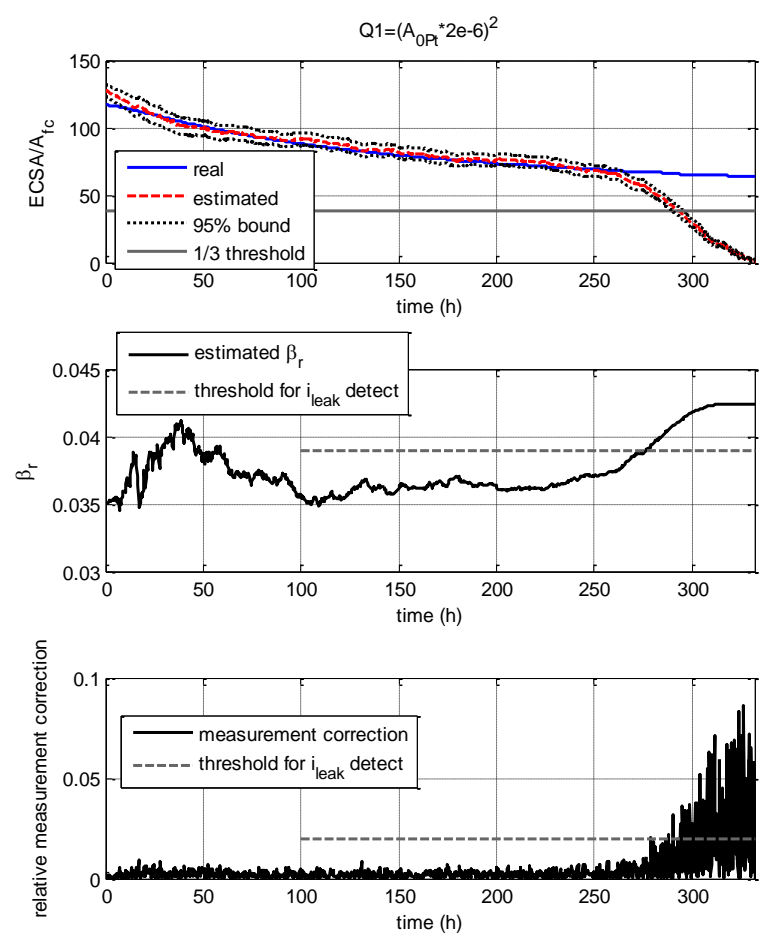

Figure 19 Simulation results for the damage tracking of ECSA and detection of current leak

\section{Conclusion}

Prognostic-oriented aging models are created to describe the slowly-varying dynamics in the fuel cell that characterize the degradation process of the MEA of the fuel cell. The ECSA and the membrane gas crossover are chosen as aging parameters based on the experiment results. The focus of this paper is the development a prognostic-oriented catalyst degradation model. Physics principles of the potential driven Ostwald ripening process are explored to establish a first-step detailed catalyst aging model. Model analysis is then conducted on this model to provide insight as for further simplification of the model for prognostic purpose. The modeling approach employed to build the degradation model for the catalyst can be extended for other materials.

An UKF-based framework is proposed for the health monitoring and prognostic scheme and applied to solve the problems. The outcome of the prognosis scheme provides information about the precision and accuracy of long-term prediction, RUL expectations and $95 \%$ confidence intervals. Simulation is carried out for the validation of the proposed scheme. The results show that with measurement correction, 
the health monitoring system can successfully track the damage variable throughout the degradation process; while at any time during the aging process, the remaining useful life can be predicted with satisfactory accuracy given that future load input information is precisely known..

\section{NOMENCLATURE}

\begin{tabular}{|c|c|c|}
\hline Symbol & Unit & Description \\
\hline$k_{1}$ & $\mathrm{~mol} / \mathrm{s} / \mathrm{cm}^{2}$ & $\begin{array}{lll}\mathrm{Pt} \text { dissolution } & \text { reaction } & \text { rate } \\
\text { constant } & & \\
\end{array}$ \\
\hline$k_{2}$ & $\mathrm{~mol} / \mathrm{s} / \mathrm{cm}^{2}$ & $\begin{array}{l}\text { Pt oxidation reaction rate } \\
\text { constant }\end{array}$ \\
\hline$A_{\mathrm{fc}}$ & $\mathrm{cm}^{2}$ & Nominal fuel cell area \\
\hline$C_{\mathrm{Pt}^{2+}}^{\mathrm{ref}}$ & $\mathrm{mol} / \mathrm{L}$ & Reference concentration for $\mathrm{Pt}^{2+}$ \\
\hline$F$ & & Faraday constant \\
\hline$M_{\mathrm{Pt}}$ & $\mathrm{g} / \mathrm{mol}$ & Molecular weight \\
\hline$R$ & $\mathrm{~J} / \mathrm{mol} \cdot \mathrm{K}$ & Universal gas constant \\
\hline$U_{1}^{\theta}$ & V & $\begin{array}{l}\text { Standard equilibrium potential of } \\
\text { Pt dissolution reaction for bulk } \\
\text { platinum }\end{array}$ \\
\hline$\tilde{U}_{2}^{\theta}$ & V & $\begin{array}{l}\text { Modified standard equilibrium } \\
\text { potential of Pt oxidation reaction }\end{array}$ \\
\hline$\rho_{\mathrm{Pt}}$ & $\mathrm{g} / \mathrm{cm}^{3}$ & Density of platinum \\
\hline$\delta_{\mathrm{CL}}$ & $\mathrm{cm}$ & Catalyst layer thickness \\
\hline$\omega$ & $\mathrm{kJ} / \mathrm{mol}$ & PtO-PtO interaction coefficient \\
\hline$\Gamma_{\max }$ & $\mathrm{mol} / \mathrm{cm}^{2}$ & $\begin{array}{l}\text { Number of moles of active sites } \\
\text { per unit of platinum area }\end{array}$ \\
\hline$r_{i}$ & $\mathrm{~cm}$ & $\begin{array}{l}\text { Radius of group } i \text { of the catalyst } \\
\text { particles }\end{array}$ \\
\hline$r_{\text {avg }}$ & $\mathrm{cm}$ & $\begin{array}{l}\text { Equivalent average } \mathrm{Pt} \text { particle } \\
\text { radius (in the sense of } \mathrm{Pt}^{2+} \\
\text { concentration balance) }\end{array}$ \\
\hline$\overline{r_{\mathrm{a}}}$ & $\mathrm{cm}$ & $\begin{array}{l}\text { Average Pt particle radius (in the } \\
\text { sense of particle geometry) }\end{array}$ \\
\hline$v_{1}$ & $\begin{array}{l}\mathrm{mol} / \mathrm{s} / \mathrm{cm}^{2}(\mathrm{Pt} \\
\text { surface area })\end{array}$ & Reaction rate of Pt dissolution \\
\hline$v_{2}$ & $\begin{array}{l}\mathrm{mol} / \mathrm{s} / \mathrm{cm}^{2}(\mathrm{Pt} \\
\text { surface area })\end{array}$ & Reaction rate of Pt oxidation \\
\hline$A_{\text {geo }}$ & $\mathrm{cm}^{2}$ & $\begin{array}{l}\text { Geometric surface area of the } \\
\text { catalyst }\end{array}$ \\
\hline$C_{\mathrm{Pt}^{2+}}$ & $\mathrm{mol} / \mathrm{L}$ & $\mathrm{Pt}^{2+}$ concentration \\
\hline$\theta_{\text {vac }}^{(i)}$ & & $\begin{array}{l}\text { Fraction of the platinum surface } \\
\text { that is not covered by oxides }\end{array}$ \\
\hline$\Delta \phi_{c}$ & V & $\begin{array}{l}\text { Potential difference between the } \\
\text { electrolyte phase and the cathode } \\
\text { phase }\end{array}$ \\
\hline
\end{tabular}

\section{REFERENCES}

Alejandro, J., Arce, A., \& Bordons, C. (2007). Development and experimental validation of a PEM fuel cell dynamic model. Journal of Power Sources, 173, 310-324.
Bi, W., \& Fuller, T. F. (2008). Modeling of PEM fuel cell $\mathrm{Pt} / \mathrm{C}$ catalyst degradation. Journal of Power Sources, 178(1), 188-196. doi:10.1016/j.jpowsour.2007.12.007

Chelidze, D., \& Cusumano, J. P. (2004). A Dynamical Systems Approach to Failure Prognosis. Journal of Vibration and Acoustics, 126(1), 2. doi: $10.1115 / 1.1640638$

Chelidze, D., Cusumano, J. P., \& Chatterjee, A. (2002). A Dynamical Systems Approach to Damage Evolution Tracking, Part 1: Description and Experimental Application. Journal of Vibration and Acoustics, 124(2), 250. doi:10.1115/1.1456908

Danzer, M. A., Wilhelm, J., Aschemann, H., \& Hofer, E. P. (2008). Model-based control of cathode pressure and oxygen excess ratio of a PEM fuel cell system. Journal of Power Sources, 176(2), 515-522.

Darling, R.M., \& Meyers, J. P. (2003). Kinetic model of platinum dissolution in PEMFCs. Journal of the Electrochemical Society, 150(11), 1523-7. doi:10.1149/1.1613669

Darling, Robert M., \& Meyers, J. P. (2005). Mathematical model of platinum movement in PEM fuel cells. Journal of the Electrochemical Society, 152(1), A242A247. doi:10.1149/1.1836156

Debe, M. K., Schmoeckel, A. K., Vernstrom, G. D., \& Atanasoski, R. (2006). High voltage stability of nanostructured thin film catalysts for PEM fuel cells. Journal of Power Sources, 161(2), 1002-1011. doi:10.1016/j.jpowsour.2006.05.033

Franco, A. A., Schott, P., Jallut, C., \& Maschke, B. (2007). A Multi-Scale Dynamic Mechanistic Model for the Transient Analysis of PEFCs. Fuel Cells, 7(2), 99-117. doi:10.1002/fuce.200500204

Franco, A.A., \& Tembely, M. (2007). Transient multiscale modeling of aging mechanisms in a PEFC cathode. Journal of the Electrochemical Society, 154, B712.

Franco, Alejandro A., Coulon, R., Ferreira de Morais, R., Cheah, S. K., Kachmar, A., \& Gabriel, M. A. (2009). Multi-scale Modeling-based Prediction of PEM Fuel Cells MEA Durability under Automotive Operating Conditions (pp. 65-79). ECS. doi:10.1149/1.3210560

Franco, Alejandro A., \& Gerard, M. (2008). Multiscale Model of Carbon Corrosion in a PEFC: Coupling with Electrocatalysis and Impact on Performance Degradation. Journal of The Electrochemical Society, 155(4), B367. doi:10.1149/1.2838165

Franco, Alejandro A., Gerard, M., Guinard, M., Barthe, B., \& Lemaire, O. (2008). Carbon Catalyst-Support Corrosion in Polymer Electrolyte Fuel Cells: Mechanistic Insights (pp. 35-55). ECS. doi:10.1149/1.3002807

Holby, E.F., Shao-Horn, Y., Sheng, W. C., \& Morgan, D. (2010). New understanding of Pt surface area loss in PEMFC's: Temperature effects. In 10th Polymer Electrolyte Fuel Cell Symposium, PEFC 10 - 218th ECS Meeting, October 10, 2010 - October 15, 2010 
(Vol. 33, pp. 369-377). Las Vegas, NV, United states: Electrochemical Society Inc. doi:10.1149/1.3484535

Holby, Edward F., Sheng, W., Shao-Horn, Y., \& Morgan, D. (2009). Pt nanoparticle stability in PEM fuel cells: Influence of particle size distribution and crossover hydrogen. Energy and Environmental Science, 2(8), 865-871. doi:10.1039/b821622n

Miotti, A., Di Domenico, A., Guezennec, Y. G., \& Rajagopalan, S. (2005). Control-oriented model for an automotive PEM fuel cell system with imbedded 1+1D membrane water transport. In 2005 IEEE Vehicle Power and Propulsion Conference, VPPC, September 7, 2005 - September 9, 2005 (Vol. 2005, pp. 611-618). Chicago, IL, United states: Inst. of Elec. and Elec. Eng. Computer Society. doi:10.1109/VPPC.2005.1554622

Okada, T. (2003). Effect of Ionic contaminants. In Handbook of Fuel Cells - Fundamentals, Technology and Applications (pp. 627-646). Wiley \& Sons.

Pukrushpan, J. T., Peng, H., \& Stefanopoulou, A. G. (2004). Control-Oriented Modeling and Analysis for Automotive Fuel Cell Systems. Journal of Dynamic Systems, Measurement, and Control, 126(1), 14. doi:10.1115/1.1648308

Schmittinger, W., \& Vahidi, A. (2008). A review of the main parameters influencing long-term performance and durability of PEM fuel cells. Journal of Power Sources, $\quad 180(1), \quad 1-14$. doi:10.1016/j.jpowsour.2008.01.070

Shao-Horn, Y., Sheng, W. C., Chen, S., Ferreira, P. J., Holby, E. F., \& Morgan, D. (2007). Instability of Supported Platinum Nanoparticles in Low-Temperature Fuel Cells. Topics in Catalysis, 46(3-4), 285-305. doi:10.1007/s11244-007-9000-0

Shengsheng Zhang, Xiaozi Yuan, Haijiang Wang, Merida, W., Hong Zhu, Jun Shen, ... Jiujun Zhang. (2009). A review of accelerated stress tests of MEA durability in PEM fuel cells. International Journal of Hydrogen Energy, 34(1), 388-404. doi:10.1016/j.ijhydene.2008.10.012

Shimoi, R., Aoyama, T., \& Iiyama, A. (2009). Development of Fuel Cell Stack Durability based on Actual Vehicle Test Data: Current Status and Future Work (No. 200901-1014). Warrendale, PA: SAE International. Retrieved from http://www.sae.org/technical/papers/2009-01-1014

Soltani, M., \& Bathaee, S. M. T. (2010). Development of an empirical dynamic model for a Nexa PEM fuel cell power module. Energy Conversion and Management, 51(12), 2492-500.

Todinov, M. T. (2001). Necessary and sufficient condition for additivity in the sense of the Palmgren-Miner rule. Computational materials science, 21(1), 101-110.

Wan, E., \& Merwe, R. (n.d.). Chapter 7: The Unscented Kalman Filter. In Kalman Filtering and Neural Networks. Wiley Publishing.
Zhang, X., \& Pisu, P. (2012). An Unscented Kalman Filter Based Approach for the Health-Monitoring and Prognostics of a PEM Fuel Cell (Vol. 3). Presented at the Annual Conference of the Prognostics and Health Management Society 2012, Minneapolis, MN.

\section{BIOGRAPHIES}

Xian Zhang received his B.S. and M.S. degrees in Automotive Engineering from Tsinghua University, Beijing, China, in 2005 and 2007, respectively, and received his Ph.D. degree in Automotive Engineering from Clemson University, Clemson, SC in August 2012. His Ph.D. research area is in the control, diagnostics and prognostics of the PEMFC system. From September 2007 to May 2012, he was a Graduate Research Assistant with the International Center for Automotive Research and Department of Automotive Engineering, Clemson University. From June to November 2010, he was an intern with National Transportation Research Center (NTRC), at Oak Ridge National Lab. Xian Zhang is a student member of ASME and SAE.

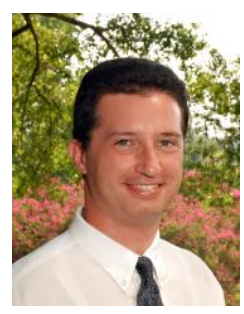

Pierluigi Pisu is an Assistant Professor at the Department of Automotive Engineering and holds a joint appointment with the Department of Electrical and Computer Engineering at Clemson University. He received his Ph.D. in Electrical Engineering from the Ohio State University in 2002. In 2004,

Dr. Pisu was granted two US patents in area of model-based fault detection and isolation. Dr. Pisu's research interests are in the area of fault diagnosis and prognosis with application to vehicle systems, energy management control of hybrid electric vehicles, vehicle to grid interaction, and systems aging. He also worked in the area of sliding mode and robust control. Dr. Pisu is member of IEEE, ASME and SAE. He is member of the IEEE Technical Committee in Automotive Control, IFAC Technical Committee in Automotive Control, Associate Editor of the Conference Editorial Board of the IEEE Control Systems Society, and Associate Editor of the SAE Journal on Alternative Powertrains. 